\title{
Performance Analysis of Planar Nanocavities-Based Wavelength Demultiplexer for Optical Communication Systems
}

\author{
Rasha H. Mahdi, R. S. Fyath \\ M.Sc Searcher, College of Engineering, Alnahrain University, Baghdad, Iraq \\ rashahmahdi@yahoo.com \\ Professor, College of Engineering, Alnahrain University, Baghdad, Iraq \\ rsfyath@yahoo.com
}

\section{ABSTRACT}

This paper presents the design and analysis of planar plasmonic wavelength demultiplexer for optical communication systems. The demultiplexer is based on silver-air-silver plasmonic waveguide supported by two nanocavities for each drop channel. One cavity is used as a resonant tunneling-based channel drop filter while the other is used to realize wavelength-selective reflection feedback in the bus waveguide. For each channel, a parametric study is performed to characterize the performance of the two corresponding nanocavities when they are operating in isolated mode. The results are then used as a basis to design a single-channel demultiplexer by introducing the coupling between the two nanocavities. Simulation results are reported for a three-channel demultiplexer $(1550,1300$, and $850 \mathrm{~nm})$ using Computer Simulation Technology (CST) software package. A drop efficiency as high as $88 \%$ is obtained for each drop channel in the designed demultiplexer. The simulated results can be used as a guideline to design ultra-compact wavelength-division multiplexing (WDM) systems in highly integrated optical circuits.

\section{Indexing terms/Keywords}

Surface plasmon polaritons (SPPs), Plasmonic waveguide, Plasmonic nanocavities, Plasmonic demultiplexer, Wavelength-division multiplexing (WDM), Multi-channel filter, Metal-Insulator structure, and Optical communication systems.

\section{Academic Discipline And Sub-Disciplines}

Electronics and Data Communications.

\section{SUBJECT CLASSIFICATION}

Optical communications.

\section{TYPE (METHOD/APPROACH)}

Research work on plasmonic demultiplexer for optical communication system.

\section{Council for Innovative Research}

Peer Review Research Publishing System

Journal: International Journal of Computers \& Technology

Vol 11, No.5

editor@cirworld.com

www.cirworld.com, member.cirworld.com 


\section{INTRODUCTION}

The capability of plasmonic structures to confine photons in the subwavelength regime has brought new concepts into the nanophotonic technology. In fact surface plasmon polaritons (SPPs) have been suggested to act as novel digital data carriers in information processing because plasmonics offers both the compactness of electronics and the speed of optics [1]. SPPs are electromagnetic (EM) waves propagating along the metal dielectric interface with an exponentially decaying field in both sides. SPPs have been considered as one of the most promising ways to overcome the classical diffraction limits of optical devices [2,3]. Therefore various plasmonic structures have been proposed and studied, such as demultiplexers for optical communication systems [4].

Recently, the plasmonic waveguides are the most competitive options for nano-optic devices, have energy transmission in visible and near infrared wavelengths and upgrades the technology of optoelectronics [5]. Two of the well-known multilayer waveguides are insulator-metal-insulator (IMI) and metal-insulator-metal (MIM) structures. The IMI waveguide gives rise to less loss for the longer propagation distance, but fails to confine a light into subwavelength scale and, thus, is not suitable for high optical integration. The MIM waveguide has a strong confinement of light and acceptable propagation length for SPPs [6]. Numerous MIM waveguide based structures have been numerically and/or experimentally demonstrated, such as bends, splitters, Mach-Zehnder interferometers, Yshaped combiners, etc [7].

Wavelength-division multiplexing (WDM) is considered to be a key technology for enhancing the transmission capacity and the application flexibility of optical communication systems. Optical multiplexers/demultiplexers are key photonic components in WDM systems to add/drop selective channels. Much effort has recently been devoted to the development of efficient surface-plasmon-based demultiplexers suitable for nanoscale integration [5, 8-10]. Different geometrical arrangements been exploited to realize such devices with increasing interest in planar nanostructures based on arrayed plasmonic slot cavities that offer the following benefits [10]

i- $\quad$ They do not need precise control over nanoparticles or any other non-planar nanostructures.

ii- The center wavelength of each channel can be controlled by finding the proper length for the slot cavity that selects the demultiplexed wavelength.

iii- The bandwidth of the channel can be adjusted by manipulation of the coupling efficiency between the slot cavity and the bus/drop waveguide.

iv- Single band transmission for each channel can be guaranteed by choosing the proper position for the drop waveguide.

Recently, Lu et al. [9] has proposed and numerically investigated a novel kind of planar nanoscale plasmonic wavelength demultiplexing structure based on channel drop filters in MIM waveguide with reflection nanocavities. The concepts of this structure were already reported by Ren et al. [11] using photonic crystal platform. Triple-wavelength demultiplexer $(928,820$, and $712 \mathrm{~nm})$ has been investigated in [9] which offer drop efficiencies up to $70 \%$. In this paper, the structure reported in [9] is redesigned for $(1550,1300$, and $850 \mathrm{~nm})$ operation. The 1550 and $1300 \mathrm{~nm}$ wavelengths correspond to, respectively, the minimum-loss wavelength and zero-dispersion wavelength of standard single-mode fibers implemented as transmission links in optical communication systems. The design methodology for each drop channel is based on investigation the characteristics of each one of the corresponding two nanocavities embedded in the structure separately before combining them to form the demultiplexer. This methodology gives more freedom in designing the demultiplexer. A drop efficiency as high as $88 \%$ is obtained for the designed three-channel demultiplexer.

In this paper, the properties of a three-channel optical demultiplexer based on silver-air-silver plasmonic waveguide and supported by two nanocavities resonators for each channel are investigated. The reflection and transmission characteristic are numerically estimated using CST software. The results reveal that the resonance wavelength of each channel can be tuned by changing the geometric parameters of the structure such as the length and width of the nanocavities, the gap between the nanocavities and the width of the bus waveguide or the drop filter. The investigated structure could find important applications in highly integrated optical circuits in the future.

\section{THEORETICAL BACKGROUND}

The interaction of metals with (EM) radiation is largely dictated by the free conduction electrons in the metal. According to the simple Drude model, the free electrons oscillate $180^{\circ}$ out of phase relative to the driving electric field. As a consequence, most metals possess a negative dielectric constant at optical frequencies which causes, for example, a very high reflectivity. Furthermore, at optical frequencies the metal's free electron gas can sustain surface and volume charge density oscillations, called plasmon polaritons or plasmons with distinct resonance frequencies. Surface plasmon polaritons are (EM) excitations that, being coupled to surface collective oscillations of free electrons in a metal, are bound to and propagate along metal-dielectric interfaces. SPP modes are renowned for their ability to concentrate (EM) fields beyond the diffraction limit (i.e., on the nanoscale), while enhancing local field strengths by several orders of magnitude [12-14].

Consider the flat interface between a metal and a dielectric material (Fig.1). The dielectric occupies the space $(y>0)$ with positive real dielectric constant $\varepsilon_{r 2}$ while the metal occupies the conducting half space $(y<0)$ and has a dielectric function $\varepsilon_{r 1}(\omega)$. Let a plane wave propagating in the $x y$ plane where the complex magnitudes of the fields are dependent on the coordinates $x$ and $y$, but constant along the $z$ direction. 


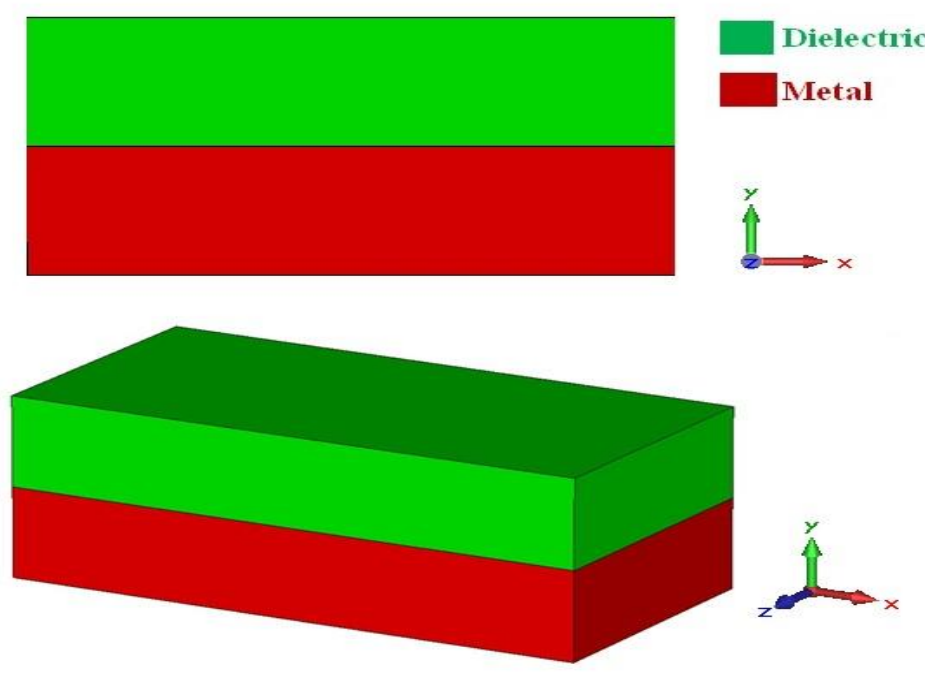

Fig. 1: Geometry for SPP propagation at a single interface between a metal and a dielectric.

In plasmonics, it is common to deal with structures without any external current sources and/or charges. In this case, Maxwell's equations of macroscopic electromagnetism take the following form [14]

$$
\begin{aligned}
& \nabla \cdot \mathbf{D}=0 \\
& \nabla \cdot \mathbf{B}=0 \\
& \nabla \times \mathbf{E}=-\frac{\partial \mathbf{B}}{\partial t} \\
& \nabla \times \mathbf{H}=\frac{\partial \mathbf{D}}{\partial t}
\end{aligned}
$$

where $\mathbf{D}$ is the dielectric displacement, $\mathbf{B}$ is the magnetic induction, $\mathbf{E}$ is the electric field, and $\mathbf{H}$ is the magnetic field.

The presence of medium modifies the (EM) fields by the following constitutive (material) equations

$$
\begin{aligned}
& \mathbf{D}=\varepsilon_{r} \mathbf{E} \\
& \mathbf{B}=\mu_{r} \mathbf{H}
\end{aligned}
$$

Here, $\varepsilon_{r}$ is the relative (electrical) permittivity (or dielectric constant), $\mu_{r}$ is the relative (magnetic) permeability, $\varepsilon_{o}$ and $\mu_{o}$ are the permittivity and permeability of free space, respectively. Assuming linear, isotropic and homogeneous material, $\varepsilon=\varepsilon_{o} \varepsilon_{r}$ and $\mu=\mu_{o} \mu_{r}$ are scalar quantites independent of the position $r$.

The boundary conditions which describe the continuity of the components of the fields perpendicular and parallel to the interface, also the momentum, can be stated as follows [13]

$$
\begin{aligned}
& \mathrm{D}_{2 y}=\mathrm{D}_{1 y} \\
& \mathrm{~B}_{2 y}=\mathrm{B}_{1 y} \\
& \mathrm{E}_{2 x}=\mathrm{E}_{1 x} \\
& \mathrm{H}_{2 x}=\mathrm{H}_{1 x} \\
& \mathrm{k}_{2 x}=\mathrm{k}_{1 x}
\end{aligned}
$$

Note that the momentum (wave number) parallel to the interface $\mathbf{k}_{x}$ is also continuous.

By application vector calculus to Maxwell's equations, one can deduce wave equations to describe the propagation of (EM) wave in terms of its electric and magnetic fields [13,14]

$$
\begin{aligned}
& \nabla^{2} \mathbf{E}-\varepsilon \mu \frac{\partial^{2}}{\partial t^{2}} \mathbf{E}=0 \\
& \nabla^{2} \mathbf{H}-\varepsilon \mu \frac{\partial^{2}}{\partial t^{2}} \mathbf{H}=0
\end{aligned}
$$

Consider harmonic time dependent of the electric field of the form

$\mathbf{E}(\mathbf{r}, t)=\mathbf{E}(\mathbf{r}) e^{-i \omega t}$

Then Eqns.(4a) and (4b) reduce to Helmholtz equations 
$\nabla^{2} \mathbf{E}(\mathbf{r})+k^{2} \mathbf{E}(\mathbf{r})=0$

$\nabla^{2} \mathbf{H}(\mathbf{r})+k^{2} \mathbf{H}(\mathbf{r})=0$

where $k=n k_{o}=\sqrt{\varepsilon_{r} \mu_{r}} k_{o}$ is the wave number, $n$ is the refractive index, and $k_{o}$ is the free space wave number. The complex amplitudes of the electric and magnetic fields can be expressed as

$\mathbf{E}(x, y, z)=\mathbf{E}(y) e^{i k_{\mathrm{x}} x}$

$\mathbf{H}(x, y, z)=\mathbf{H}(y) e^{i \mathrm{k}_{\mathrm{x}} x}$

The complex parameter $\mathrm{k}_{\mathrm{x}}$ is called the propagation constant of the traveling waves and corresponds to the component of the wave vector in the direction of propagation. Inserting these expressions into Eqn. (6a) and (6b), respectively yields the form of the wave equation [14]

$\frac{\partial^{2} \mathbf{E}(y)}{\partial y^{2}}+\left(k_{o}^{2} \varepsilon_{r} \mu_{r}-\mathrm{k}_{\mathbf{x}}^{2}\right) \mathbf{E}(\mathbf{y})=0$

$\frac{\partial^{2} \mathbf{H}(y)}{\partial y^{2}}+\left(k_{o}^{2} \varepsilon_{r} \mu_{r}-\mathrm{k}_{\mathbf{x}}^{2}\right) \mathbf{H}(y)=0$

Expressions for the different field components of $\mathbf{E}$ and $\mathbf{H}$ can be achieved in a startforward way using the curl Equations (1c) and (1d), and for harmonic time dependence $\left(\frac{\partial}{\partial t}=-i \omega\right)$, propagation along the $x$-direction $\left(\frac{\partial}{\partial x}=i \mathrm{k}_{\mathrm{x}}\right)$, and homogeneity in the $z$-direction $\left(\frac{\partial}{\partial z}=0\right)$, this system of equations simplifies to [12-14]

$\frac{\partial \mathrm{E}_{\mathrm{z}}}{\partial y}=-i \omega \mu_{o} \mu_{r} \mathrm{H}_{x}$

$\frac{\partial \mathrm{E}_{\mathrm{x}}}{\partial y}-i \mathrm{k}_{\mathrm{x}} \mathrm{E}_{y}=i \omega \mu_{o} \mu_{r} \mathrm{H}_{\mathrm{z}}$

$i \mathrm{k}_{\mathrm{x}} \mathrm{E}_{z}=i \omega \mu_{o} \mu_{r} \mathrm{H}_{y}$

$\frac{\partial \mathrm{H}_{\mathrm{z}}}{\partial y}=i \omega \varepsilon_{o} \varepsilon_{r} \mathrm{E}_{x}$

$\frac{\partial \mathrm{H}_{\mathrm{x}}}{\partial y}-i \mathrm{k}_{\mathrm{x}} \mathrm{H}_{y}=-i \omega \varepsilon_{o} \varepsilon_{r} \mathrm{E}_{z}$

$i \mathrm{k}_{\mathrm{x}} \mathrm{H}_{z}=-i \omega \varepsilon_{o} \varepsilon_{r} \mathrm{E}_{y}$

Due to the linearity of these equations, one can divide the problem of a wave incident to a planar surface into two parts depending on the polarization of light: modes transverse electric (TE or s-modes) and transverse magnetic (TM or $p$ modes) [13]. These modes are depicted in Fig. 2 where the plane of incidence is defined as the $x y$ plane. The following results are valid for nonmagnetic materials (i.e., $\mu_{r 1}=\mu_{r 2}=1$ ) which are usually adopted in conventional plasmonic structures.

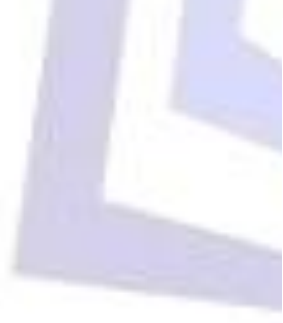

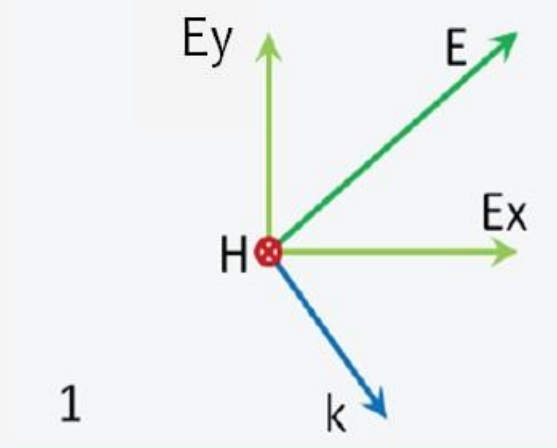

2
P-Polarised wave

$$
\begin{aligned}
& \mathbf{H}_{x}, \mathbf{H}_{y}, \mathbf{E}_{z}=0 \\
& \mathbf{E}_{x}, \mathbf{E}_{y}, \mathbf{H}_{z} \neq 0
\end{aligned}
$$

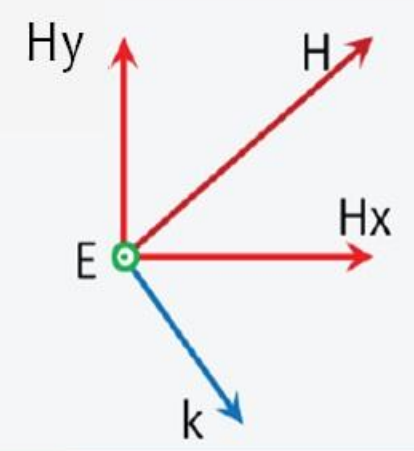

S-Polarised wave

$$
\begin{aligned}
& \mathbf{H}_{x}, \mathbf{H}_{y}, \mathbf{E}_{z} \neq 0 \\
& \mathbf{E}_{x}, \mathbf{E}_{y}, \mathbf{H}_{z}=0
\end{aligned}
$$

Fig. 2: Interface between two media (1 and 2), showing the polarisation vectors of a p-polarised (left) and an spolarised waves (right). The plane of incidence coincides with the page. A p-polarised wave (TM) is characterised by the magnetic field perpendicular to the plane of incidence; while an s-polarised (TE) wave is characterised by the electric field perpendicular to the plane of incidence [13]. 


\section{A. TE modes}

Here only $\mathrm{H}_{x}, \mathrm{H}_{y}$ and $\mathrm{E}_{z}$ being nonzero and the set of equations reduces to

$\mathrm{H}_{x}=i \frac{1}{\omega \mu_{o}} \frac{\partial \mathrm{E}_{\mathrm{z}}}{\partial y}$

$\mathrm{H}_{y}=\frac{\mathrm{k}_{\mathrm{x}}}{\omega \mu_{o}} \mathrm{E}_{z}$

with the TE wave equation

$\frac{\partial^{2} \mathrm{E}_{\mathrm{z}}}{\partial y^{2}}+\left(k_{o}{ }^{2} \varepsilon_{r}-\mathrm{k}_{\mathrm{x}}^{2}\right) \mathrm{E}_{\mathrm{z}}=0$

Using Eqns. (10a-10c), the respective expressions for the field components are [12,13]

For $(\boldsymbol{y}>0)$

$\mathrm{H}_{x}(y)=-i A_{2} \frac{1}{\omega \mu_{o}} k_{y 2} e^{i \mathrm{k}_{\mathrm{x}} x} e^{-k_{y 2} y}$

$\mathrm{H}_{y}(y)=A_{2} \frac{\mathrm{k}_{\mathrm{x}}}{\omega \mu_{o}} e^{i \mathrm{k}_{\mathrm{x}} x} e^{-k_{y 2} y}$

$\mathrm{E}_{z}(y)=A_{2} e^{i \mathrm{k}_{\mathrm{x}} x} e^{-k_{y 2} y}$

For $(\boldsymbol{y}<0)$

$\mathrm{H}_{x}(y)=i A_{1} \frac{1}{\omega \mu_{o}} k_{y 1} e^{i \mathrm{k}_{\mathrm{x}} x} e^{k_{y 1} y}$

$\mathrm{H}_{y}(y)=A_{1} \frac{\mathrm{k}_{\mathrm{x}}}{\omega \mu_{o}} e^{i \mathrm{k}_{\mathrm{x}} x} e^{k_{y 1} y}$

$\mathrm{E}_{z}(y)=A_{1} e^{i \mathrm{k}_{\mathrm{x}} x} e^{k_{y 1} y}$

Where $k_{y i}(i=1,2)$ is the component of the wave vector perpendicular to the interface in the two media. Continuity of $\mathrm{E}_{z}$ and $\mathrm{H}_{x}$ at the interface leads to the condition

$A_{1}\left(k_{y 1}+k_{y 2}\right)=0$

Since confinement to the surface requires $\operatorname{Re}\left[k_{y 1}\right]>0$ and $\operatorname{Re}\left[k_{y 2}\right]>0$, this condition is only fulfilled if $A_{1}=0$, so that also $A_{2}=A_{1}=0$ [12].

\section{B. TM modes}

Here only the field components $\mathrm{E}_{x}, \mathrm{E}_{y}$ and $\mathrm{H}_{\mathrm{z}}$ are nonzero

$\mathrm{E}_{x}=-i \frac{1}{\omega \varepsilon_{o} \varepsilon_{r}} \frac{\partial \mathrm{H}_{\mathrm{z}}}{\partial y}$

$\mathrm{E}_{y}=-\frac{\mathrm{k}_{\mathrm{x}}}{\omega \varepsilon_{o} \varepsilon_{r}} \mathrm{H}_{z}$

and the wave equation for TM modes is

$\frac{\partial^{2} \mathrm{H}_{\mathrm{z}}}{\partial y^{2}}+\left(k_{o}{ }^{2} \varepsilon_{r}-\mathrm{k}_{\mathrm{x}}{ }^{2}\right) \mathrm{H}_{\mathrm{z}}=0$

Using Eqns. (13a-13c) in both half spaces yields [12,13]

For $(\boldsymbol{y}>0)$

$\mathrm{E}_{x}(y)=i A_{2} \frac{1}{\omega \varepsilon_{0} \varepsilon_{r 2}} k_{y 2} e^{i \mathrm{k}_{\mathrm{x}} x} e^{-k_{y 2} y}$

$\mathrm{E}_{y}(y)=-A_{1} \frac{\mathrm{k}_{\mathrm{x}}}{\omega \varepsilon_{o} \varepsilon_{r 2}} e^{i \mathrm{k}_{\mathrm{x}} x} e^{-k_{y 2} y}$

$\mathrm{H}_{z}(y)=A_{2} e^{i \mathrm{k}_{\mathrm{x}} x} e^{-k_{y_{2}} y}$

For $(\boldsymbol{y}<0)$

$\mathrm{E}_{x}(y)=-i A_{1} \frac{1}{\omega \varepsilon_{o} \varepsilon_{r 1}} k_{y 1} e^{i \mathrm{k}_{\mathrm{x}} x} e^{k_{y 1} y}$

$\mathrm{E}_{y}(y)=-A_{1} \frac{\mathrm{k}_{\mathrm{x}}}{\omega \varepsilon_{o} \varepsilon_{r 1}} e^{i \mathrm{k}_{\mathrm{x}} x} e^{k_{y 1} y}$

$\mathrm{H}_{z}(y)=A_{1} e^{i \mathrm{k}_{\mathrm{x}} x} e^{k_{y 1} y}$

Continuity of $\mathrm{H}_{\mathrm{z}}$ and $\varepsilon_{r i} E_{y}$ at the interface requires that $A_{1}=A_{2}$ and 
$\frac{k_{y 2}}{k_{y 1}}=-\frac{\varepsilon_{r 2}}{\varepsilon_{r 1}}$

Note that with the convention of the signs in the exponents in Eqns. (14), confinement to the surface demands Re $\left[\varepsilon_{r 1}\right]<0$ if $\varepsilon_{r 2}>0$ - the surface waves exist only at interfaces between materials with opposite signs of the real part of their dielectric permittivities, i.e., between a conductor and an insulator [12]. The expression for $\mathrm{H}_{\mathrm{z}}$ has to fulfill the wave Eqn. (13c), yielding $[12,14]$

$k_{y 1}^{2}=\mathrm{k}_{\mathrm{x}}^{2}-k_{o}^{2} \varepsilon_{r 1}$

$k_{y 2}^{2}=\mathrm{k}_{\mathrm{x}}{ }^{2}-k_{o}^{2} \varepsilon_{r 2}$

Using Eqn. (15) yields the dispersion relation of SPPs propagating at the interface between the two half spaces

$\mathrm{k}_{\mathrm{x}}=k_{o} \sqrt{\frac{\varepsilon_{r 1} \varepsilon_{r 2}}{\varepsilon_{r 1}+\varepsilon_{r 2}}}$

\section{SINGLE-CHANNEL DEMULTIPLEXER}

This section presents results related to $1550 \mathrm{~nm}$ demultiplexer. Illustrative results related to $1300 \mathrm{~nm}$ and $850 \mathrm{~nm}$ demultiplexers are given in Appendices' A and B. respectively.

\subsection{Device structure and theoretical model}

A three-dimensional (3D) Ag-air-Ag MIM demultiplexer is analyzed using CST software. The plasmonic demultiplexer is composed of one bus waveguide and two nanocavities, one is used for a resonant tunneling-based channel drop filter (cavity 1) while the other is used to realize wavelength-selective reflection feedback in the bus waveguide (cavity 2), see Fig. 3 [9]. The drop waveguide can be sufficiently reinforced by setting a proper distance between the drop and reflection cavities $(D)$.

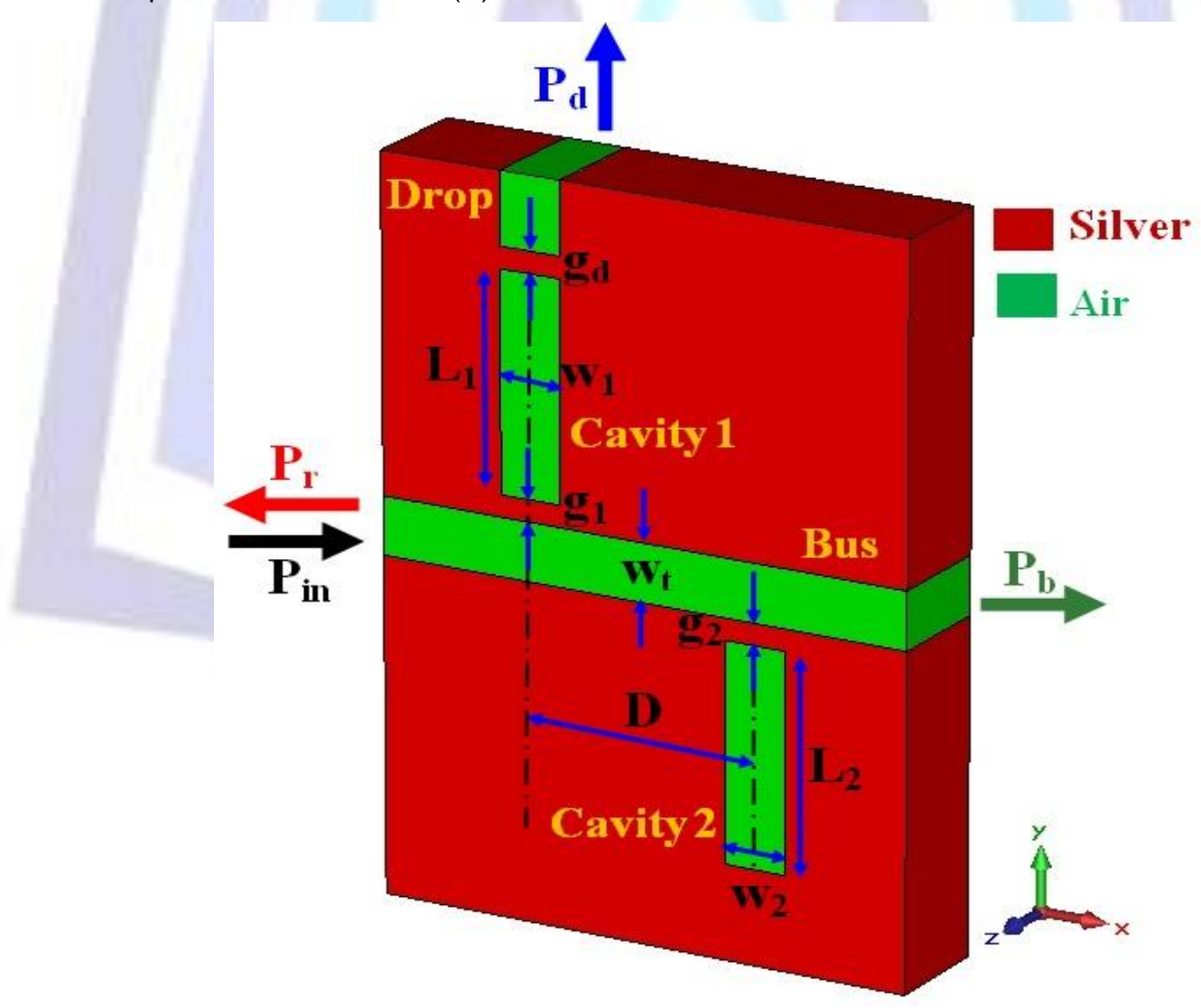

Fig. 3: Schematic diagram of 3D MIM plasmonic demultiplexer consists of a drop nanocavity coupled with a feedback nanocavity.

The dielectric used in the slits and cavities is air (the relative dielectric constant $\varepsilon_{d}=1$ ) while the metal is assumed as silver. The frequency-dependent complex relative permittivity of silver $\varepsilon_{m}(f)$ is characterized by the well-known Drude mode [15] 
$\varepsilon_{m}(f)=\varepsilon_{\infty}-\frac{f_{p}^{2}}{[f(f+i \gamma)]}$

where $\varepsilon_{\infty}=3.7$ is the dielectric constant at infinite frequency, $f_{p}=2200 \mathrm{THz}$ stands for the bulk plasma frequency, $\gamma=4.353 \mathrm{THz}$ is the electron collision frequency, and $f$ is the frequency of the incident (EM) radiation in vacuum [4].

Since the width of the bus waveguide is much smaller than the incident wavelength $\lambda$, only the fundamental TM mode is excited in the structure, whose dispersion relation is governed by equations [16]

$\varepsilon_{m} \sqrt{n_{e f f}^{2}-\varepsilon_{d}} \tanh \left[\frac{w_{t} k_{o} \sqrt{n_{e f f}^{2}-\varepsilon_{d}}}{2}\right]+\varepsilon_{d} \sqrt{n_{e f f}^{2}-\varepsilon_{m}}=0$

Where $k_{o}=2 \pi / \lambda$ is the free-space wave vector and $n_{\text {eff }}$ denotes the effective refractive index (ERI) of the SPP mode. Note that $n_{e f f}$ is related to the wavelength $\lambda$ and the width $w_{t}$ of the bus waveguide. The phase between the two reference planes can be expressed as $\varphi=D \beta_{s p p}=D n_{\text {effr }} k_{o}$, where $\beta_{s p p}$ is the propagation constant of the SPP waves in the MIM waveguide and $n_{\text {effr }}$ represents the real part of the (ERI) of the bus waveguide.

One can expect the nanocavity to operate as a resonator. Resonance waves can be formed only in some appropriate conditions within nanocavity segment. Defining $\Delta \emptyset$ to be the phase delay per round-trip in the nanocavity, one has $\Delta \emptyset=4 \pi n_{\text {effr }} L / \lambda+2 \emptyset_{r}$ where $\emptyset_{r}$ is the phase shift of the SPPs reflected at one facet of the cavity and $L$ is the cavity length. Obviously, only when the resonant condition $\Delta \emptyset=2 m \pi$ is satisfied, the waves propagating through the structure will be trapped within the nanocavity and hence the resonance wavelength can be obtained. The parameter $m$ is a positive integer, which stands for the number of antinodes of the standing SPP waves [17]. Thus, the resonance wavelength of the MIM cavity can be derived as [8]

$\lambda_{r}=\frac{2 n_{e f f r} L}{\left(m-\emptyset_{r} / \pi\right)}$

One can see from Eqn. (20) that the resonant wavelength $\lambda_{r}$ is proportional to the length and the effective index of the cavity. Obviously, only the waves with the wavelength $\lambda_{r}$ can exist stably in the nanocavity, and thus partly transmitted through the output end of the nanocavity.

\subsection{Simulation results}

\section{A. Effective refractive index (ERI)}

The starting point is to calculate the (ERI) $n_{\text {eff }}$ in the air slot waveguide. The results are obtained by solving Eqn. (19) numerically at $\lambda=1550 \mathrm{~nm}$ and for different values of slot width $w$. Figure 4(a) shows the variation of the real and imaginary parts, $n_{\text {effr }}$ and $n_{\text {effi }}$, with slot width. At $w=50 \mathrm{~nm}, n_{\text {eff }}=1.375+j 0.0038$. The imaginary part of the (ERI) determines the attenuation introduced by the waveguide. The amplitude (field) attenuation constant $\alpha_{a}=2 \pi n_{\text {effi }} / \lambda$ and the power attenuation coefficient in (decibel per unit length) $\alpha_{d B}=8.68 \alpha_{a}$. The dependence of the waveguide attenuation $\alpha_{d B}$ on the slot width is depicted in Fig. 4(b). The result reveal that $\alpha_{d B}=0.135 \mathrm{db} / \mathrm{um}$ when $=50 \mathrm{~nm}$.
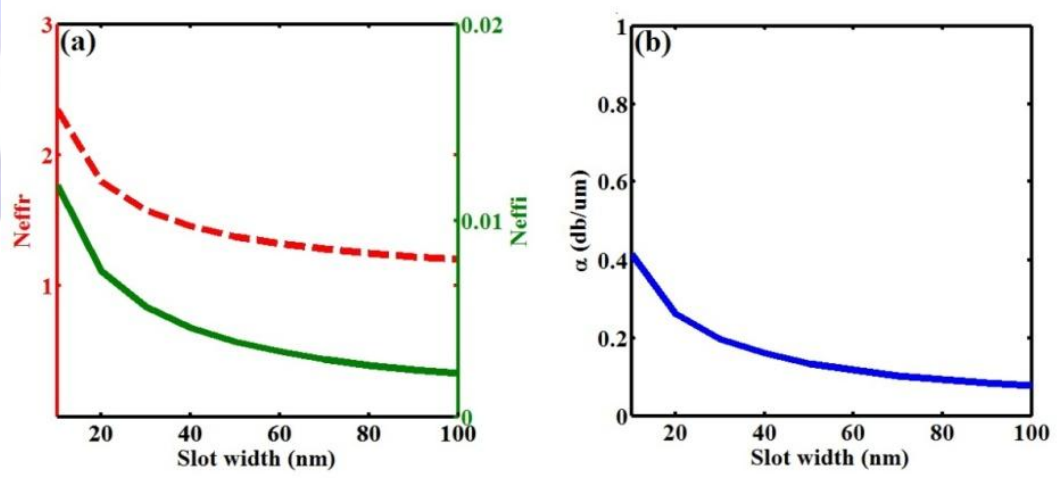

Fig. 4: (a) Variation of the real and imaginary parts of (ERI) with air slot width at $\lambda=1550 \mathrm{~nm}$. (b) dependence of the waveguide attenuation on the width.

\section{B. Drop cavity (cavity 1)}

The tunneling-based channel drop filter (cavity 1 ) is designed according to the structure shown in Fig. 5, with bus width $w_{t}=50 \mathrm{~nm}$ and cavity slot width $w_{1}=50 \mathrm{~nm}$. The distance between the cavity and the bus waveguide $g_{1}$ is set to 7 $\mathrm{nm}$ while the distance between the cavity and the drop waveguide $g_{d}$ is set to $8 \mathrm{~nm}$. The cavity length $L_{1}$ is tuned in the region of $\lambda / 2 n_{\text {effr }}$ to ensure oscillation with $m=1$ (see Eqn. (20)). 


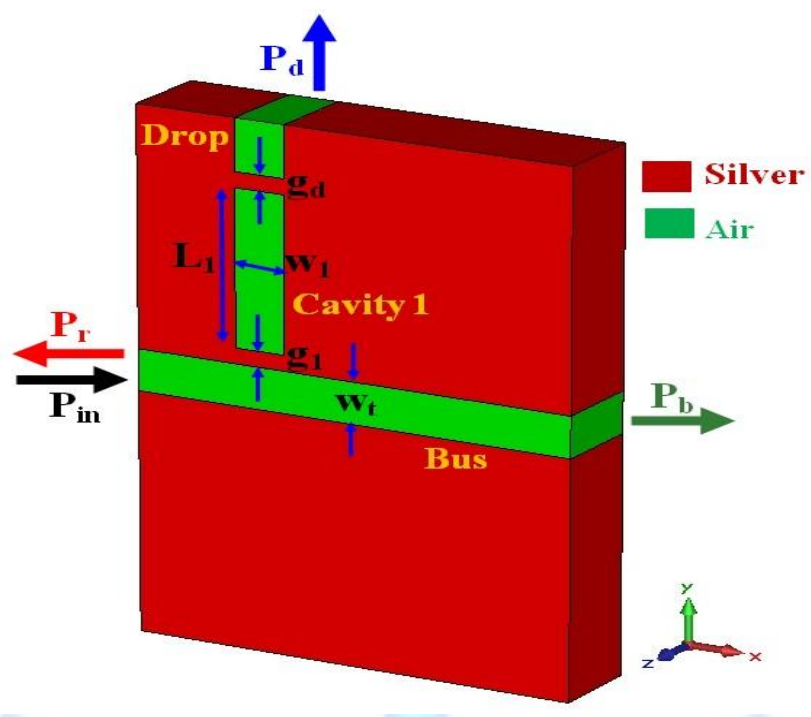

Fig. 5: Schematic diagram of 3D MIM plasmonic waveguide with a drop nanocavity (cavity 1).

Figure 6(a) shows the power spectra corresponding to the transmission coefficient through the drop port $T_{d} \equiv$ $P_{d} / P_{i n}$, the transmission coefficient at the bus output $T_{b} \equiv P_{b} / P_{\text {in }}$ and the reflection coefficient at the input port $T_{r} \equiv P_{r} / P_{i n}$, for $L_{1}=492 \mathrm{~nm}$. At $\lambda=1550 \mathrm{~nm}, T_{d}=18.9 \%, T_{b}=55.9 \%$ and $T_{r}=3.9 \%$. Hence $T_{d}+T_{b}+T_{r}=78.7 \%$, which indicates a power loss of $1.04 \mathrm{~dB}$. Figure $6(\mathrm{~b})$ shows the field distribution $\left|\mathrm{H}_{z}\right|$ at $\lambda=1550 \mathrm{~nm}$.

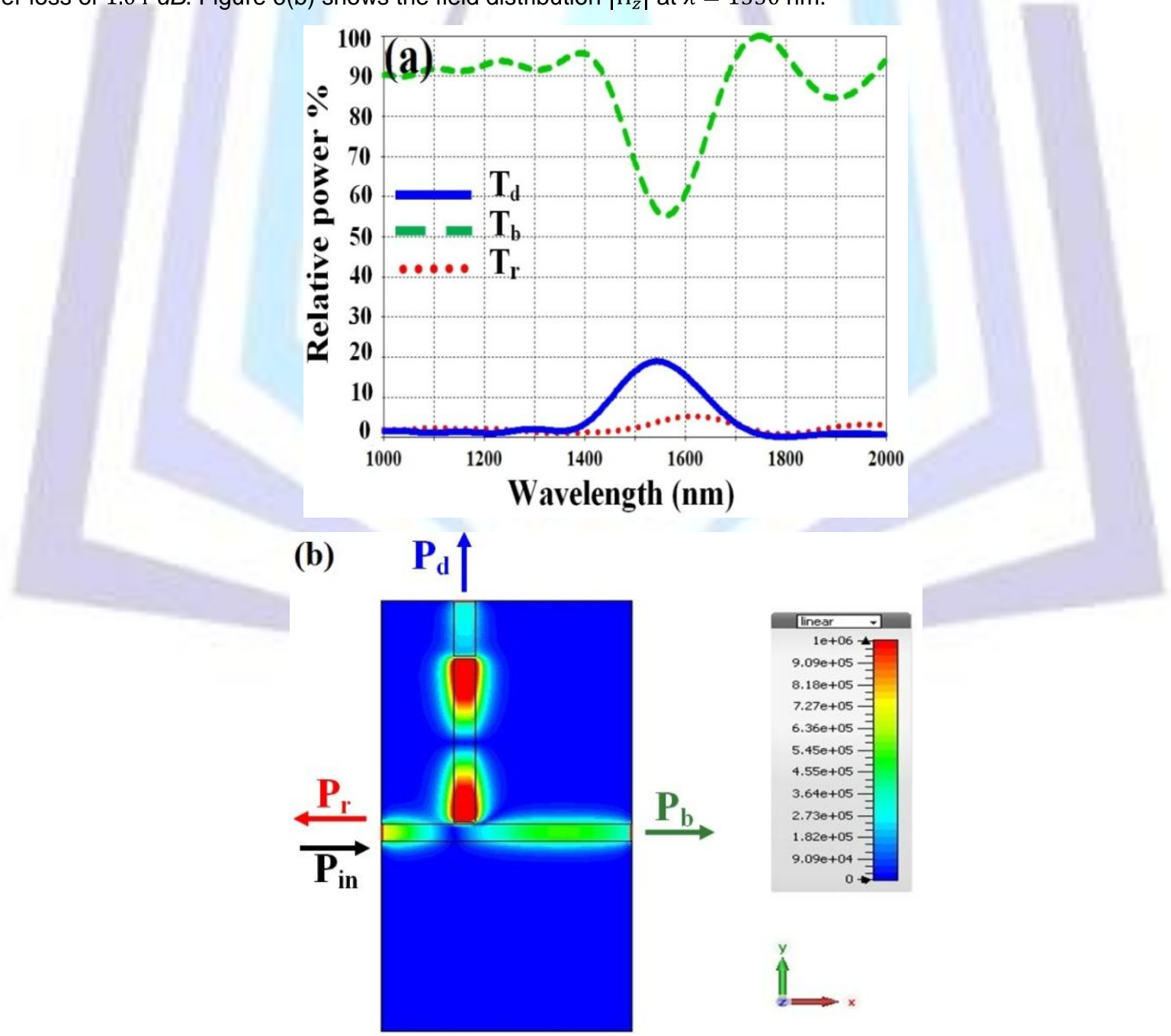

Fig. 6: (a) Power spectra associated with cavity 1. (b) Field distribution of $\left|H_{z}\right|$ with incident wavelength of 1550 nm. 
The variation of the resonance wavelength $\lambda_{r}, 3 \mathrm{~dB}$ bandwidth $\Delta \lambda$ associated with the spectrum of the power transmission coefficients at the drop port, the quality factor $Q_{o}=\lambda_{r} / \Delta \lambda$, and power reflected and transmission coefficients at different ports are plotted in figure 7. Investigated the results in Figure 7(a) reveals that the resonance wavelength $\lambda_{r}$ increases linearly with cavity length $L_{1}$ (as expected from Eqn. (20)). The desired $\lambda=1550 \mathrm{~nm}$ is achieved when $L_{1}=492$ $\mathrm{nm}$. Figure 7 (b) highlight the fact that $\Delta \lambda$ varies almost linearly with $L_{1}$; at $1550 \mathrm{~nm}, \Delta \lambda$ is $199 \mathrm{~nm}$. Therefore, the quality factor is less sensitive to cavity length variation, Figure 7 (c). At $1550 \mathrm{~nm}, Q_{o}$ is 7.788 which is relatively low value. Figure 7(d) illustrates the effects of cavity length on the power coefficient. Note that $T_{r}<T_{d}$ and $T_{b}$. Note further that $T_{b}\left(T_{d}\right)$ increases (decreases) with cavity length.
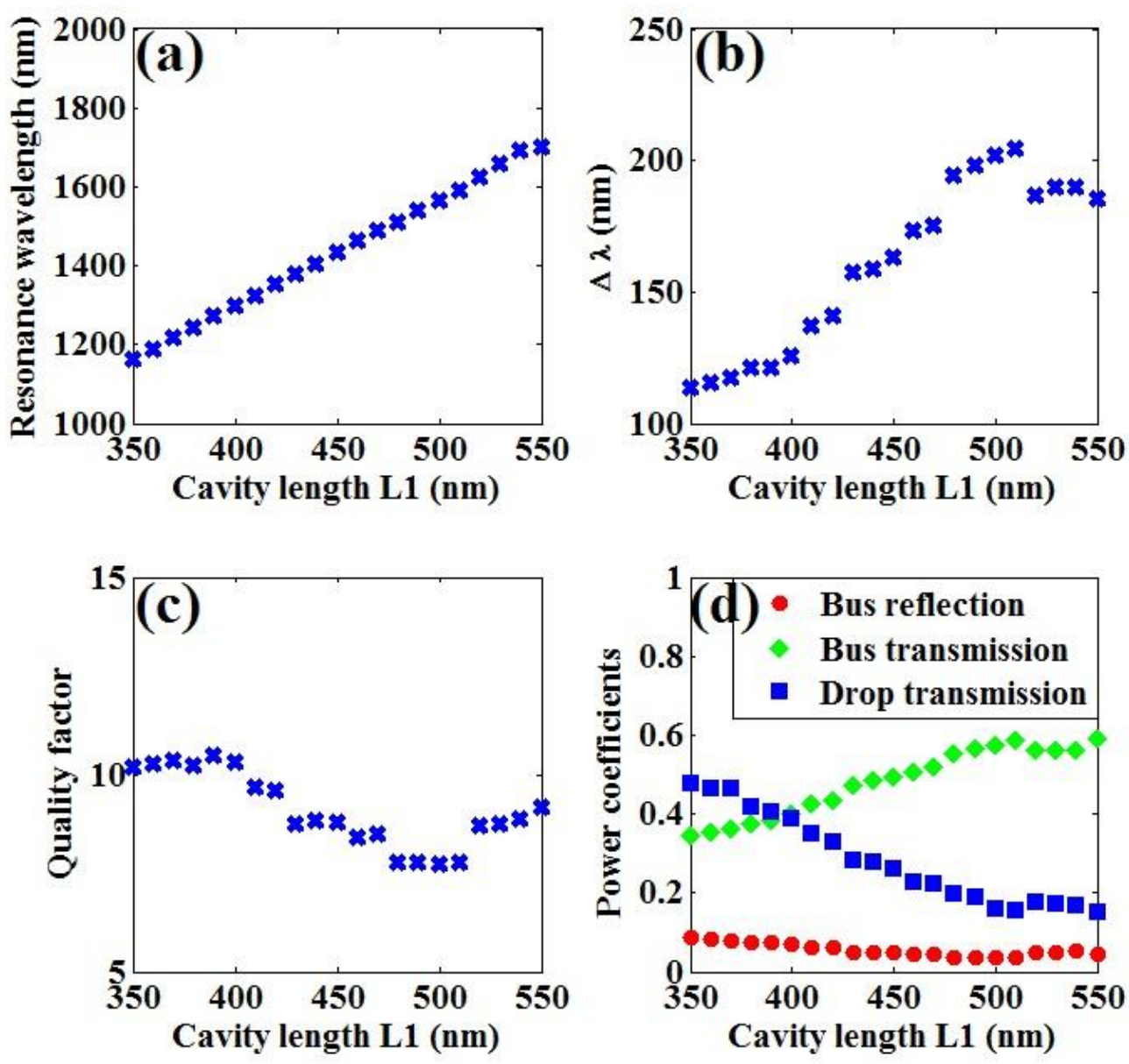

Fig. 7: Effect of cavity length $L_{1}$ on (a) resonance wavelength, (b) spectrum bandwidth, (c) quality factor, and (d) power coefficients.

The next step is to investigate the effect of the other geometric parameters on the characteristics of the cavity. Figure 8 illustrates the effect of the coupling distance $g_{1}$ when $L_{1}=492 \mathrm{~nm}$ and $g_{d}=8 \mathrm{~nm}$ The calculations are repeated in Fig. 9 to assess the effect of the coupling distance $g_{d}$ when $L_{1}=492 \mathrm{~nm}$ and $g_{1}=7 \mathrm{~nm}$. Investigating the results in Figs. 8 and 9 highlights the following facts

i. Each of the coupling distances can be used to tune the resonance wavelength. The resonance wavelength decreases almost linear with the coupling distance with $\partial \lambda_{r} / \partial g_{1}=2.75$ and $\partial \lambda_{r} / \partial g_{d}=4.1$.

ii. Both $g_{1}$ and $g_{d}$ affect strongly the reflection and transmission coefficients and therefore these coupling distances should be calculated carefully in designing the demultiplexer. Increasing $g_{1}$ from $7 \mathrm{~nm}$ to $10 \mathrm{~nm}$ yields $T_{d}=$ $12.2 \%, T_{b}=68.9 \%$ and $T_{r}=1.5 \%$. Increasing $g_{d}$ from $8 \mathrm{~nm}$ to $10 \mathrm{~nm}$ yields $T_{d}=19.2 \%, T_{b}=48.1 \%$, and $T_{r}=6.4 \%$. These values are to be compared with $T_{d}=18.9 \%, T_{b}=55.9 \%$ and $T_{r}=3.9 \%$ when $g_{1}=7 \mathrm{~nm}$ and $g_{2}=8 \mathrm{~nm}$. iii. 

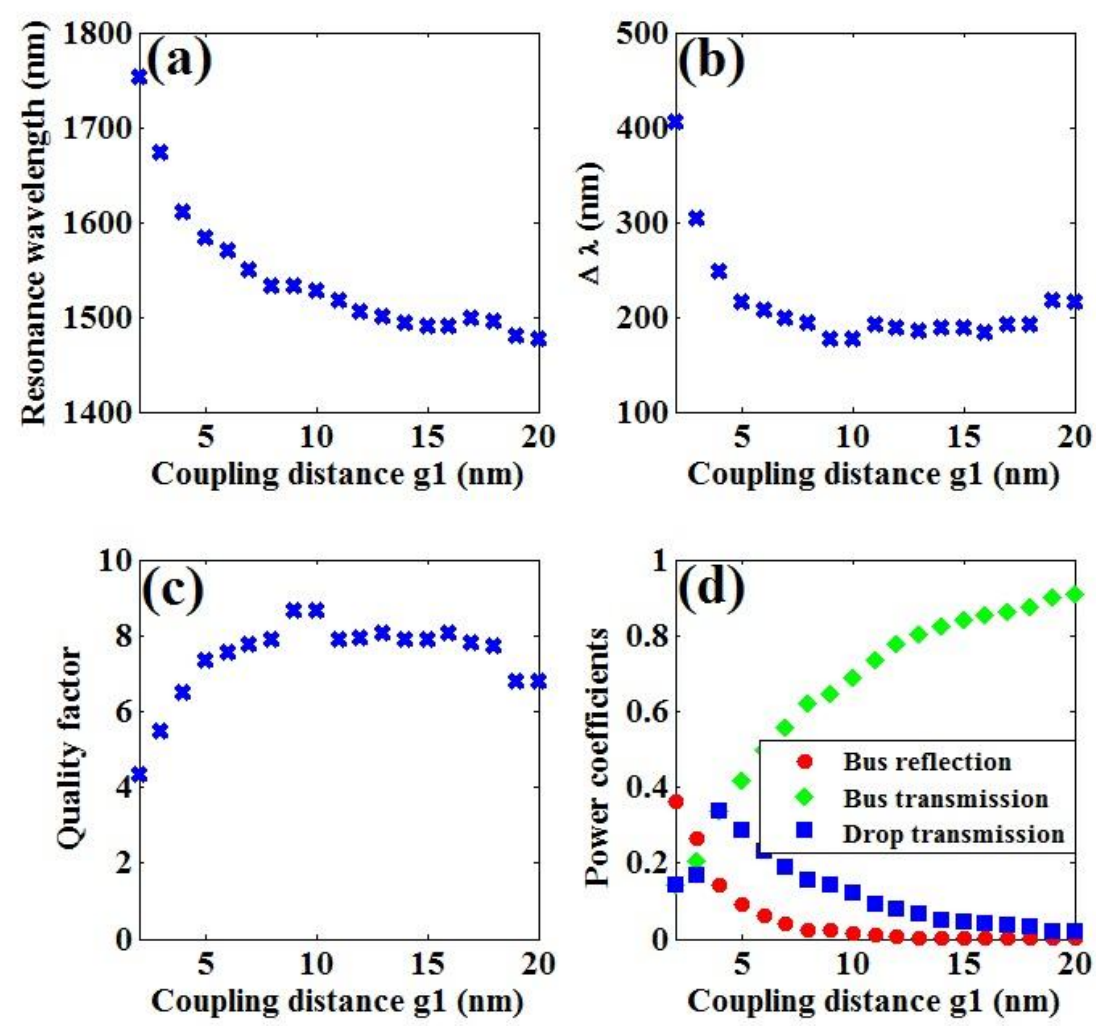

Fig. 8: Effect of cavity length $g_{1}$ on (a) resonance wavelength, (b) spectrum bandwidth, (c) quality factor, and (d) power coefficients.
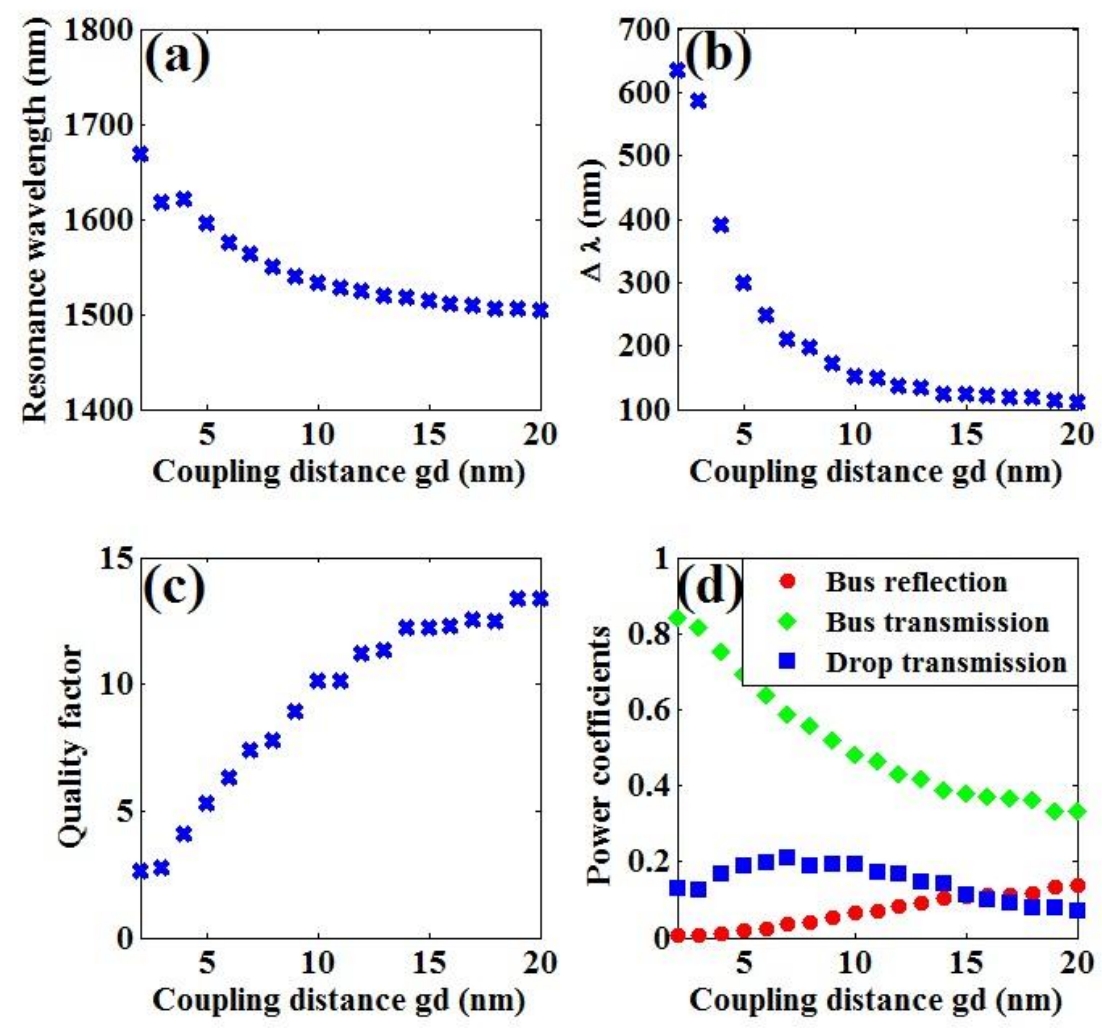

Fig. 9: Effect of cavity length $g_{d}$ on (a) resonance wavelength, (b) spectrum bandwidth, (c) quality factor, and (d) power coefficients. 
The effect of cavity width $w_{1}$ on the tuning characteristics of the cavity is also investigated and the results are depicted in Fig. 10 for $L_{1}=492 \mathrm{~nm}, g_{1}=7 \mathrm{~nm}$ and $g_{d}=8 \mathrm{~nm}$. The key role behind the effect of $w_{1}$ comes from the variation of the slot (ERI) with its width as predicted by Eqn. (19). The variation of the real part of $n_{e f f}$ with $w_{1}$ also affects the reflection phase shift per cavity facet $\emptyset_{r}$ as deduced from Eqn. (20). Investigating the results in Fig. 10 reveals the following findings

i. The resonance wavelength decreases with increasing the slot width. In fact $\lambda_{r}$ is almost linearly increasing function with $1 / w_{1}$. Appling curve fitting to the result depicted in Fig. 10(a) yields $\lambda_{r} \cong a+b / w_{1}$ with $a=1250 \mathrm{~nm}$ and $b=$ $15000 \mathrm{~nm}$. Increasing $w_{1}$ from $50 \mathrm{~nm}$ to $100 \mathrm{~nm}$ will decrease $\lambda_{r}$ from $1550 \mathrm{~nm}$ to $1391.5 \mathrm{~nm}$.

ii. The transmission bandwidth $\Delta \lambda$ is slightly sensitive to slot width variation. At $w_{1}=50 \mathrm{~nm}$ and $100 \mathrm{~nm}, \Delta \lambda=199$ and 176.8 , respectively. The quality factor is almost independent of $w_{1}$ when $w_{1}>40 \mathrm{~nm}$. The quality factor is 7.788 and 7.863 when $w_{1}$ is $50 \mathrm{~nm}$ and $100 \mathrm{~nm}$, respectively.

iii. The cavity width affects strongly the reflection and transmission coefficients. Increasing $w_{1}$ from $50 \mathrm{~nm}$ to $100 \mathrm{~nm}$ will change $T_{d}$ from $18.9 \%$ to $44.9 \%, T_{b}$ from $55.9 \%$ to $34.1 \%$ and $T_{r}$ from $3.9 \%$ to $11.3 \%$.

The phase shift introduces by reflection at one cavity facet $\emptyset_{r}$ is estimated from Eqn. (20) and the results are as given in Fig. 10(e).
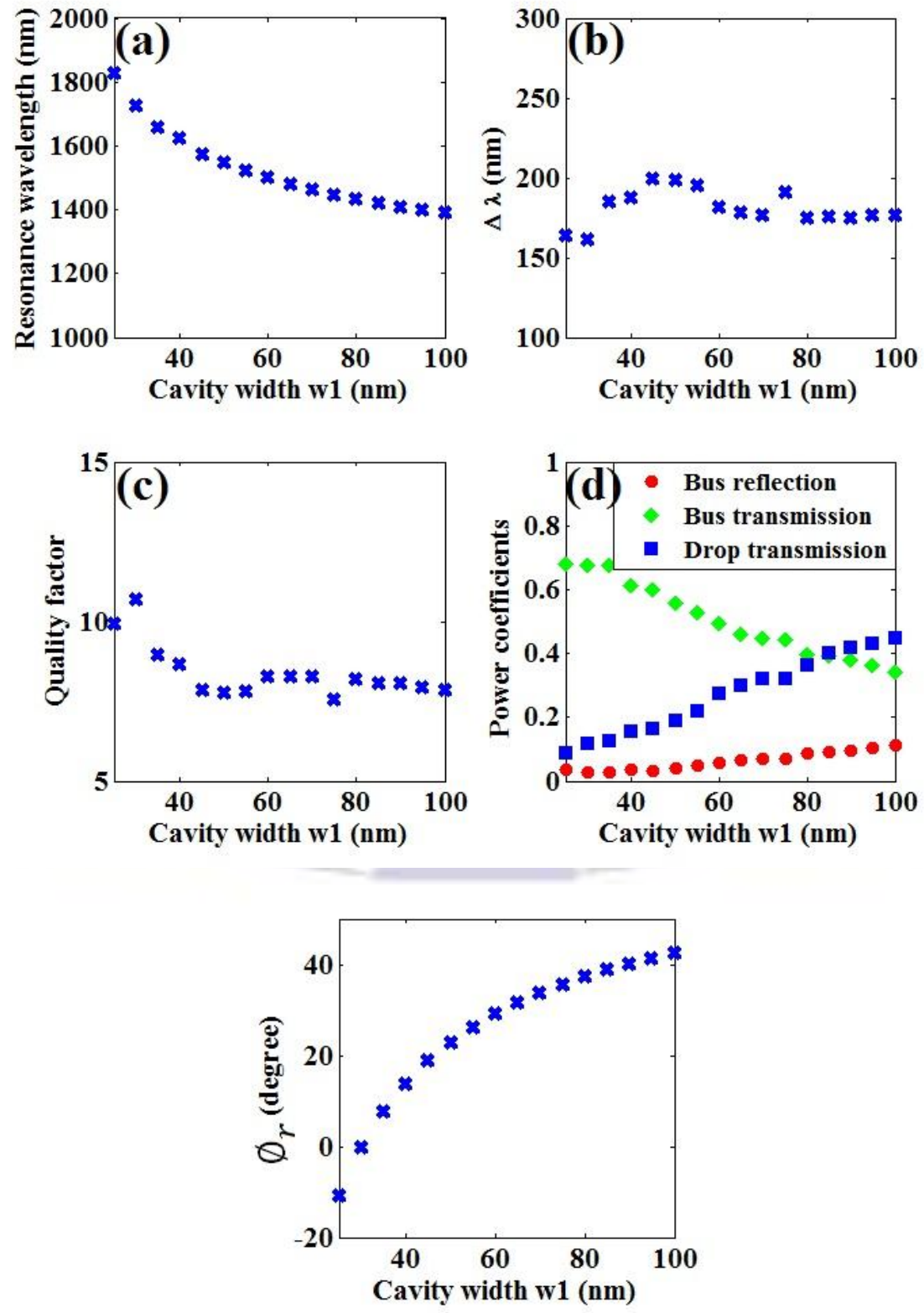

Fig. 10: Effect of cavity length $w_{1}$ on (a) resonance wavelength, (b) spectrum bandwidth, (c) quality factor, (d) power coefficients, and (e) phase shift of SPPs reflected at one facet of the cavity. 


\section{Feedback cavity (cavity 2)}

The simulation is carried further to investigate the performance of the wavelength selective reflection (feedback) cavity. The cavity is side-coupled to the bus waveguide, as shown in Fig. 11 . The widths of the bus $w_{t}$ and the cavity slot $w_{2}$ are set to $50 \mathrm{~nm}$. The distance between the cavity and the bus $g_{2}=5 \mathrm{~nm}$. The cavity length $L_{2}$ is tuned in the region of $\lambda / 2 n_{\text {effr }}$ to ensure oscillation with $m=1$ (see Eqn. (20)).

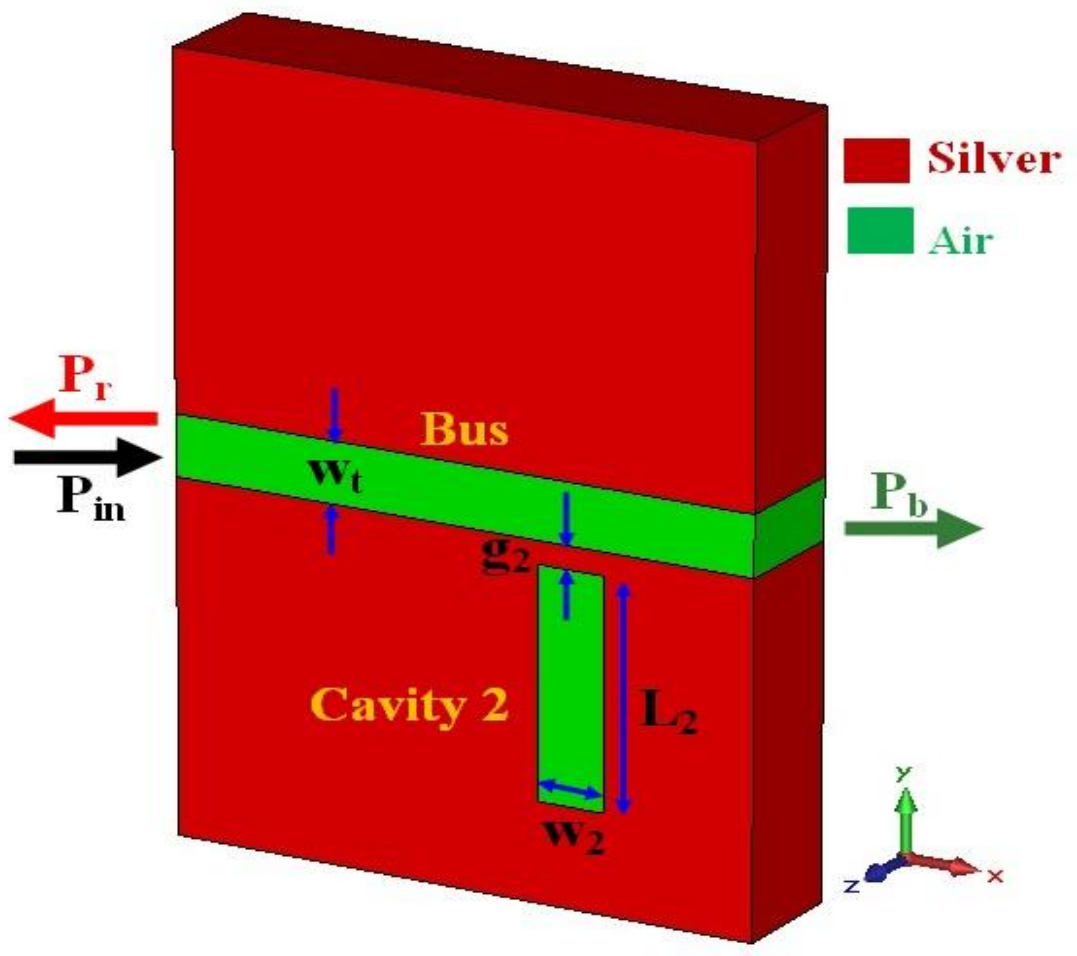

Fig. 11: Schematic diagram of 3D MIM plasmonic waveguide with a feedback cavity (cavity 2).

Figure 12(a) shows the power spectra corresponding to the transmission coefficient at the bus output $T_{b} \equiv P_{b} / P_{\text {in }}$ and the reflection coefficient at the input port $T_{r} \equiv P_{r} / P_{\text {in }}$ for $L_{1}=492 \mathrm{~nm}$. At $\lambda_{r}=1550 \mathrm{~nm}, T_{b}=47.1 \%$ and $T_{r}=13.0 \%$. Hence $T_{b}+T_{r}=60.1 \%$ which indicates a power loss of $2.21 \mathrm{db}$. Figure $12(\mathrm{~b})$ show the field distribution $\left|\mathbf{H}_{\mathbf{z}}\right|$ at $\lambda=1550$ $\mathrm{nm}$.

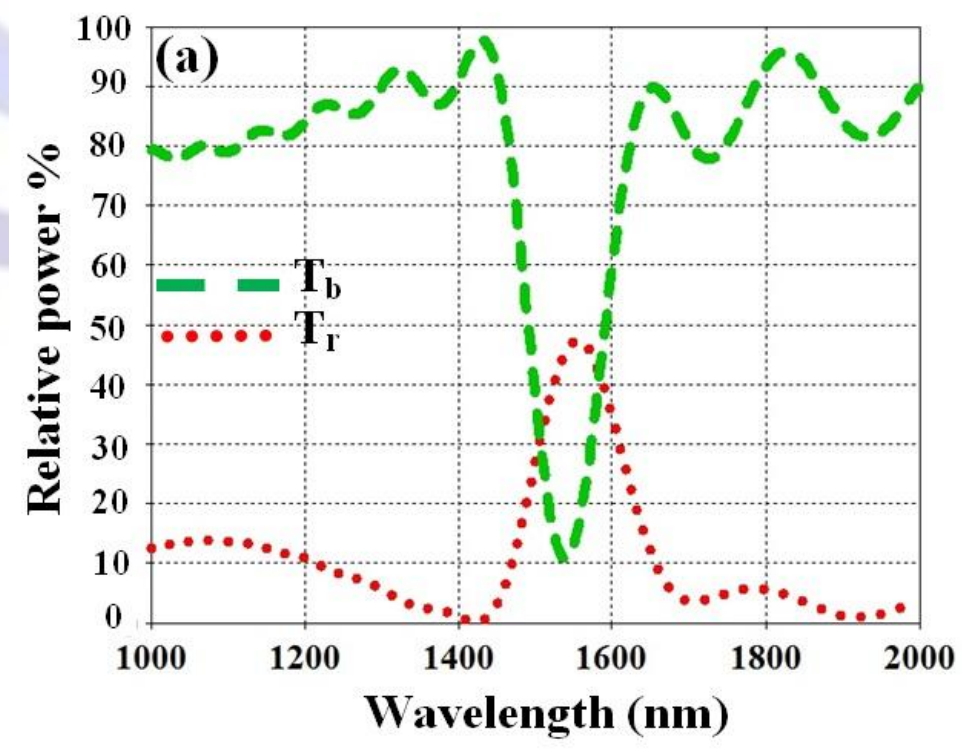




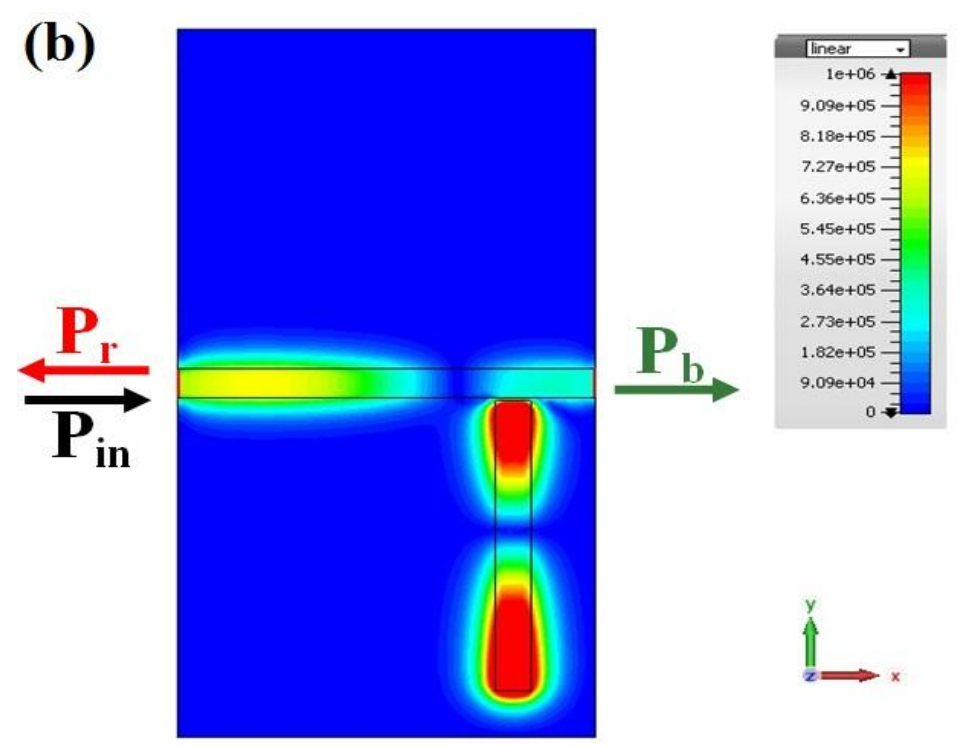

Fig. 12: (a) Power spectra associated with the cavity 2. (b) Field distribution of $\left|\mathrm{H}_{z}\right|$ with $1550 \mathrm{~nm}$-incident wavelength.

Figure 13 illustrates the effect of cavity length $L_{2}$ on the characteristics of the cavity. The results in Fig. 13(a) highlight the linear variation of the resonance wavelength $\lambda_{r}$ with $L_{2}\left(\partial \lambda_{r} / \partial L_{2}=2.57\right)$. At $L_{2}=492 \mathrm{~nm}$, the resonance wavelength equals to $1550 \mathrm{~nm}$. When $L_{2}>350 \mathrm{~nm}$, the bandwidth of the reflection spectrum changes almost linearly with $L_{2}$ (Fig. 13(b)) leading to almost constant quality factor (Fig. 13(c)). The quality factor is 12.3 when $\lambda_{r}=1550 \mathrm{~nm}$. Figure 13(d) displays the variation of the power coefficients at the bus ports with cavity length. Note that $T_{b}\left(T_{r}\right)$ increases (decreases) with cavity length; $T_{b}<T_{r}$ for the values of $L_{2}$ considered here.
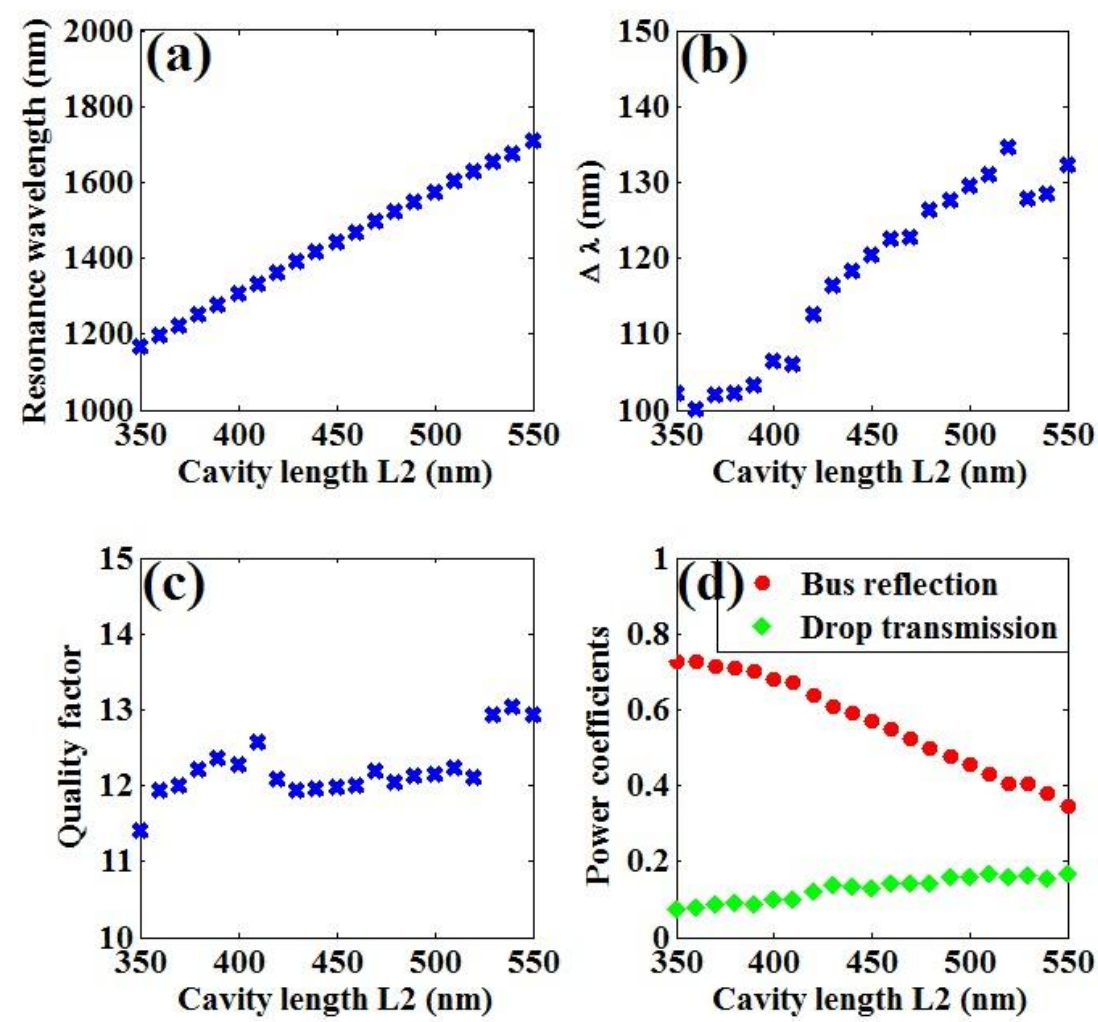

Fig. 13: Effect of cavity length $L_{2}$ on (a) resonance wavelength, (b) spectra bandwidth, (c) quality factor and (d) power coefficients.

The effect of the coupling distance $g_{2}$ on the characteristics of the cavity is also investigated and the results are depicted in Fig. 14 for $L_{2}=492 \mathrm{~nm}$. As $g_{2}$ increases, both $\lambda_{r}$ and $\Delta \lambda$ decrease while the quality factor increases almost linearly with slop $\partial \lambda_{r} / \partial g_{2}=7.7$. Figure $14(\mathrm{~d})$ illustrates clearly that $T_{b}$ and $T_{r}$ are affected strongly when $g_{2}$ varies. The 
increase of $g_{2}$ reduces the feedback effect of the cavity leading to a an increase in $T_{b}$ and decrease in $T_{r}$. Increasing $g_{2}$ from $5 \mathrm{~nm}$ to $10 \mathrm{~nm}$ will increase $T_{b}$ from $13.0 \%$ to $38.2 \%$ and decrease $T_{r}$ from $47.0 \%$ to $19.3 \%$.
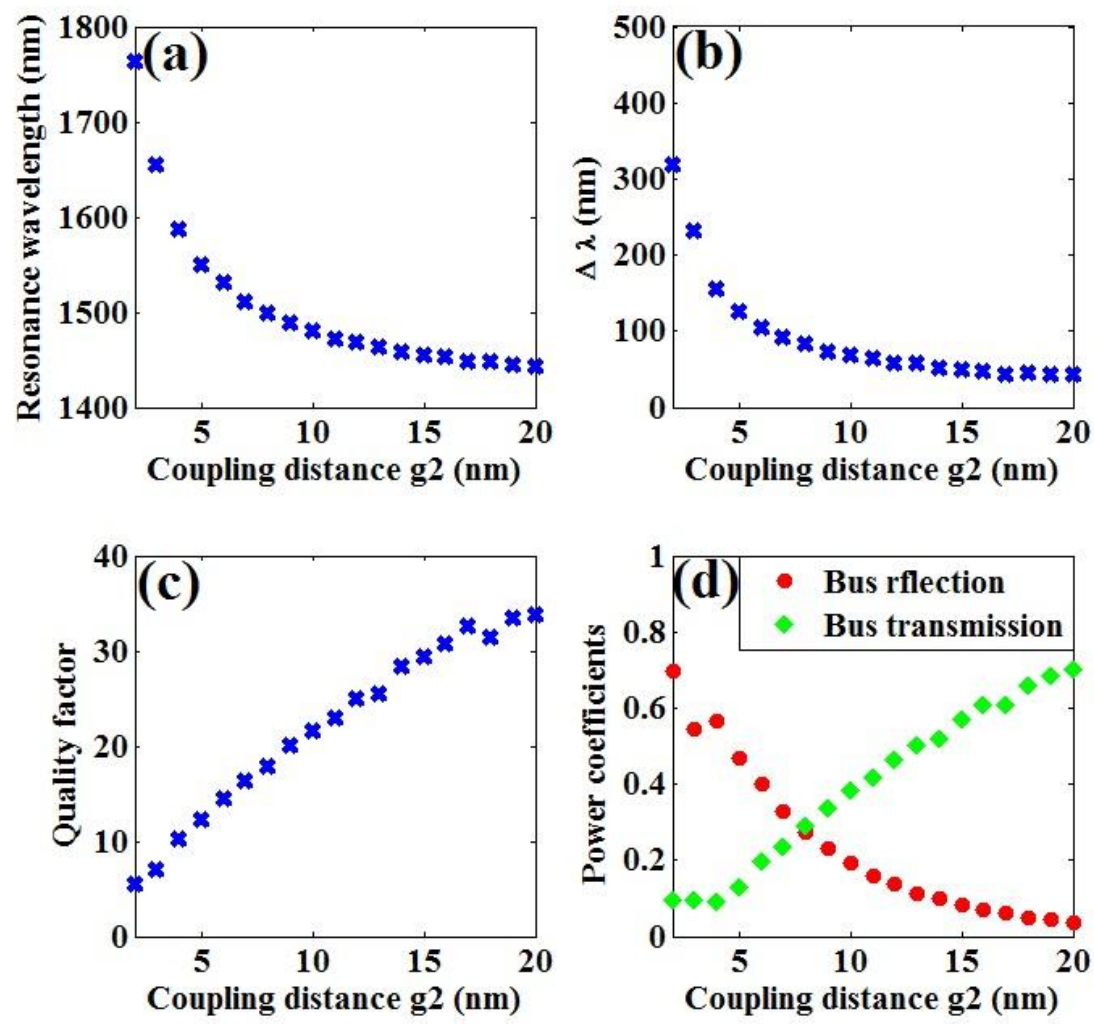

Fig. 14: Effect of cavity length $g_{2}$ on (a) resonance wavelength, (b) spectrum bandwidth, (c) quality factor, and (d) power coefficients.

Figure 15 illustrates the effect of slot width $w_{2}$ on the cavity characteristics. The resonance wavelength decreases with $w_{2}$ and approaches $1460.3 \mathrm{~nm}$ at $w_{2}=75 \mathrm{~nm}$. The transmission and reflection coefficients are affected strongly by $w_{2}$ variation (see Fig. 15(d)). $T_{b}$ and $T_{r}$ approach $10.7 \%$ and $64.6 \%$, respectively, when $w_{2}=75 \mathrm{~nm}$. These values are to be compared with $13.0 \%$ and $47.1 \%$, respectively, for $w_{2}=50 \mathrm{~nm}$.
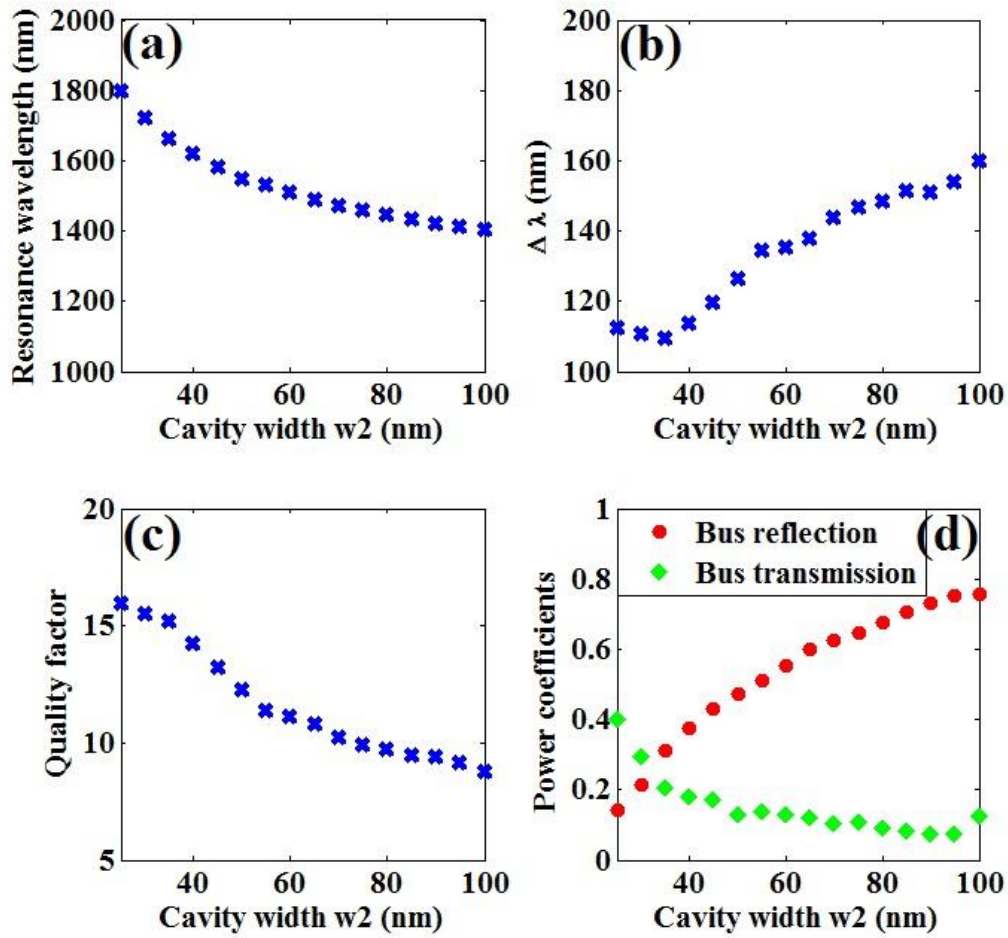


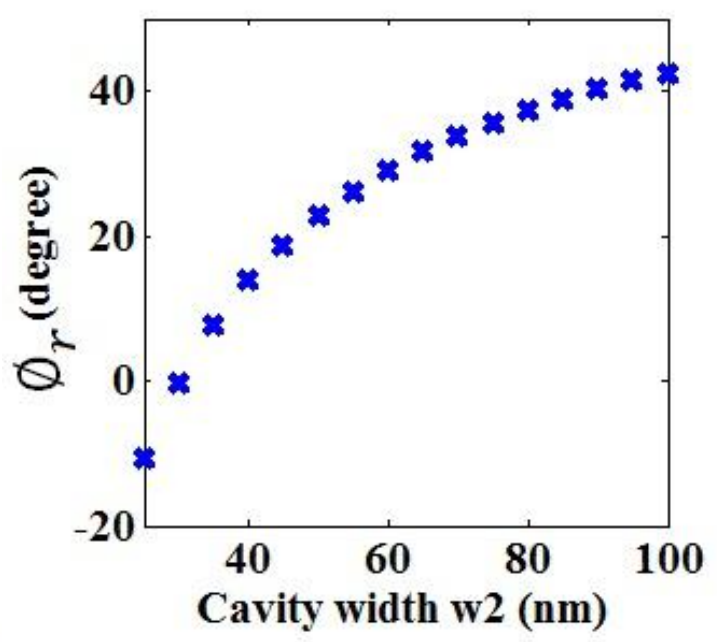

Fig. 15: Effect of cavity length $w_{2}$ on (a) resonance wavelength, (b) spectrum bandwidth, (c) quality factor, (d) power coefficients, and (e) phase shift of SPPs reflected at one facet of the cavity.

\section{Demultiplexer characteristics}

The structure of the plasmonic demultiplexer in Fig. 3 is designed for $1550 \mathrm{~nm}$-demultiplexing using the following geometric parameters (deduces from the previous subsections) $w_{t}=w_{1}=w_{2}=50 \mathrm{~nm}, L_{1}=L_{2}=492 \mathrm{~nm}, g_{1}=7 \mathrm{~nm}$, $g_{d}=8 \mathrm{~nm}$ and $g_{2}=5 \mathrm{~nm}$. The distance $D$ between the reference planes of the two cavities is tuned during the simulation with best results are obtained with $D=281 \mathrm{~nm}$.

Figure 16(a) illustrates the power spectra of transmission coefficient at the drop port $T_{d}$, transmission coefficient at the bus output port $T_{b}$ and the reflection coefficient at the input port $T_{r}$. At $\lambda=1550 \mathrm{~nm}, T_{d}$ approaches its peak value $87.8 \%$ while $T_{b}$ approaches its minimum value $2.0 \%$ and the reflection coefficient $T_{r}$ is $1.3 \%$. The bandwidth of the transmission spectrum $T_{d}$ is $13.45 \mathrm{~nm}$ which gives $Q=14.4 \mathrm{~nm}$. The successful operation of the demultiplexer at $\lambda=1550$ $\mathrm{nm}$ is depicted in Fig. 16(b) which shows the field distribution $\left|\mathrm{H}_{z}\right|$.

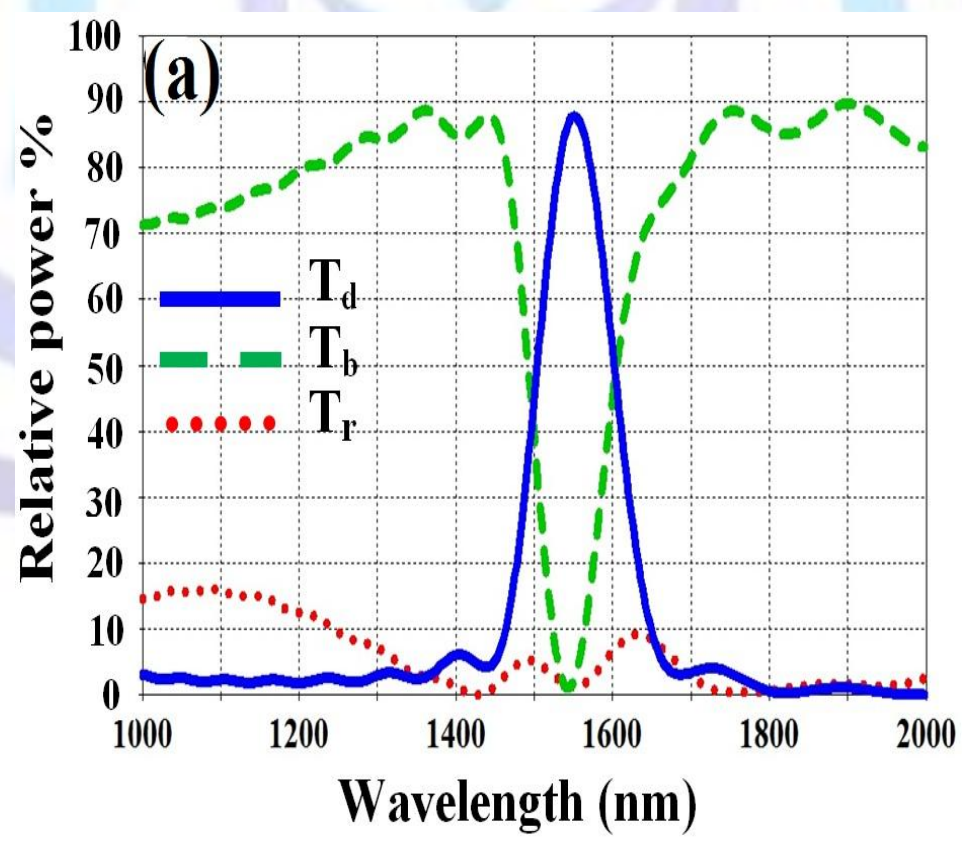

Fig. 16: Power spectra associated with the $1550 \mathrm{~nm}$ demultiplexer. (b) field distribution of $\left|\mathrm{H}_{z}\right|$ with incident wavelength $1550 \mathrm{~nm}$. 


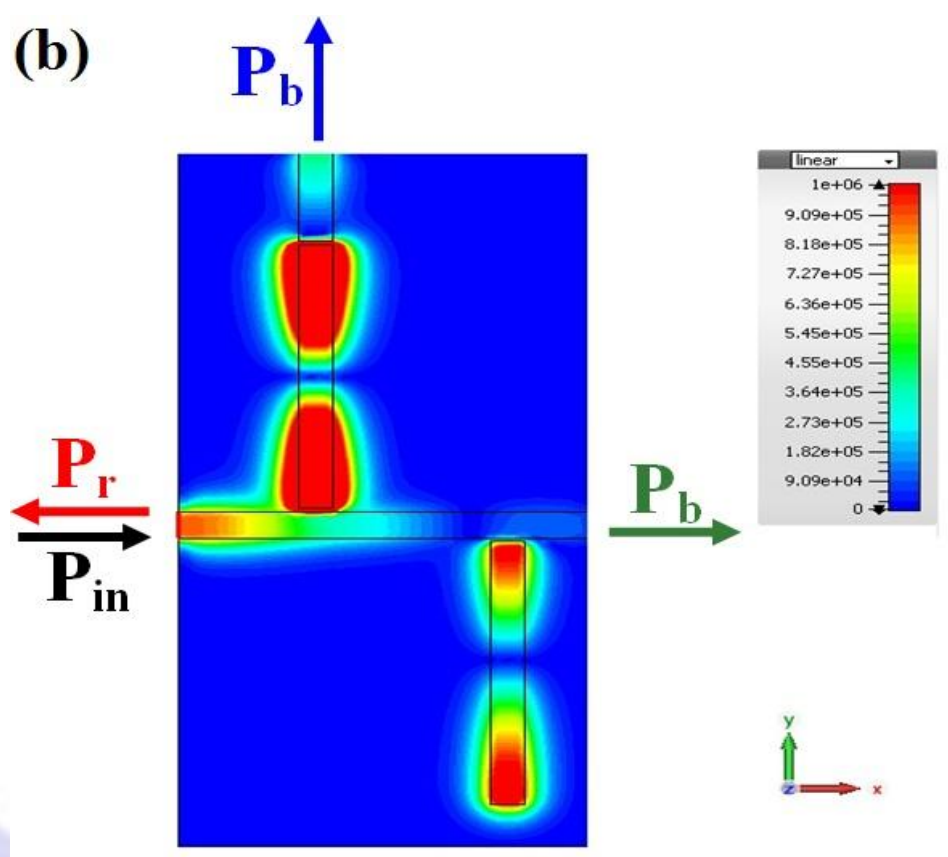

Fig. 16: (Continued).

\section{THREE-CHANNEL DEMULTIPLEXER}

The results reported in Section 3 and Appendices $A$ and $B$ are used as a guide line to design a three-channel demultiplexer for the wavelengths 1550,1300 and $850 \mathrm{~nm}$ (see Fig. 17). The geometric parameters of each channel are slightly tuned to reduce the effect of coupling between the channels and hence enhancing the drop efficiency at the corresponding output port (see Table 1).

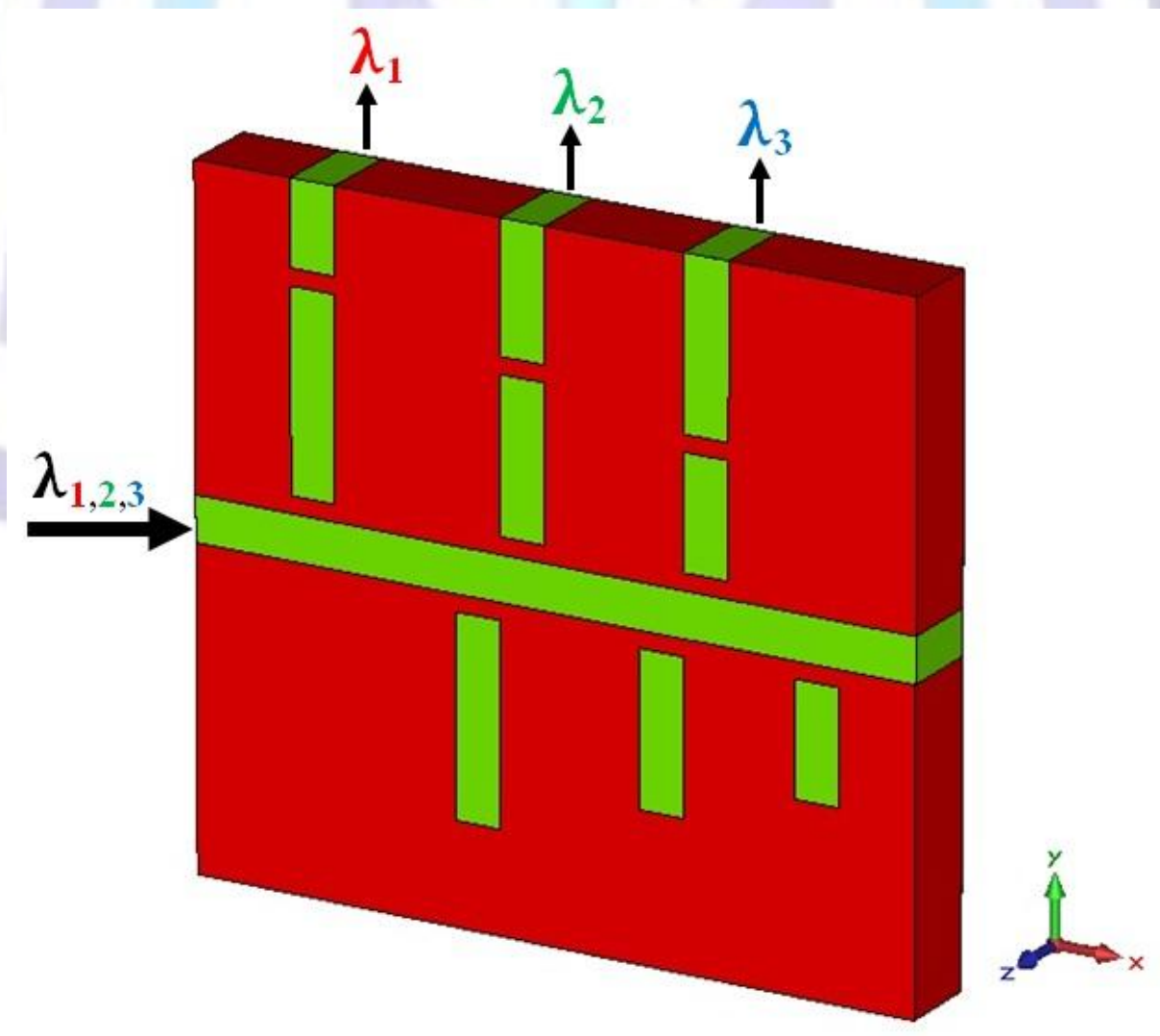

Fig. 17: Schematic diagram of 3D MIM plasmonic demultiplexer of three-channel demultiplexer for the wavelengths 1550, 1300 and $850 \mathrm{~nm}$ 
Table 1. Goemetric parameters for the $(1550,1300$, and $850 \mathrm{~nm})$ demultiplexer.

\begin{tabular}{|c|c|c|c|}
\hline \multirow{2}{*}{$\begin{array}{c}\text { Wavelength } \\
(\mathbf{n m})\end{array}$} & $\begin{array}{c}\text { Geometric } \\
\text { parameters } \\
(\mathbf{n m})\end{array}$ & \multicolumn{2}{|c|}{ Values (nm) } \\
\cline { 3 - 4 } & Initial & Final \\
\hline $\mathbf{1 5 5 0}$ & $L_{1}=L_{2}$ & 492 & 491 \\
\hline & $g_{1}$ & 7 & 7 \\
\hline & $g_{2}$ & 5 & 5 \\
\hline & $g_{d}$ & 8 & 7 \\
\hline $\mathbf{1 3 0 0}$ & $L_{1}=L_{2}$ & 406 & 409 \\
\hline & $g_{1}$ & 7 & 10 \\
\hline & $g_{2}$ & 6 & 6 \\
\hline $\mathbf{8 5 0}$ & $g_{d}$ & 10 & 8 \\
\hline & $L_{1}=L_{2}$ & 255 & 246 \\
\hline & $g_{1}$ & 12 & 8 \\
\hline & $g_{2}$ & 8 & 7 \\
\hline & $g_{d}$ & 13 & 14 \\
\hline
\end{tabular}

Figure 18(a) shows the spectrum of the power drop efficiency of the thee-channel demultiplexer. Drop efficiencies of $87.99 \%, 86.35 \%$, and $88.02 \%$ are obtaine at 1550,1300 , and $850 \mathrm{~nm}$. The distribution of the field component $H_{z}$ across the structure is shown in Figs. 18(b-d) when the device is excited by 1550, 1300 and $850 \mathrm{~nm}$, respectively.

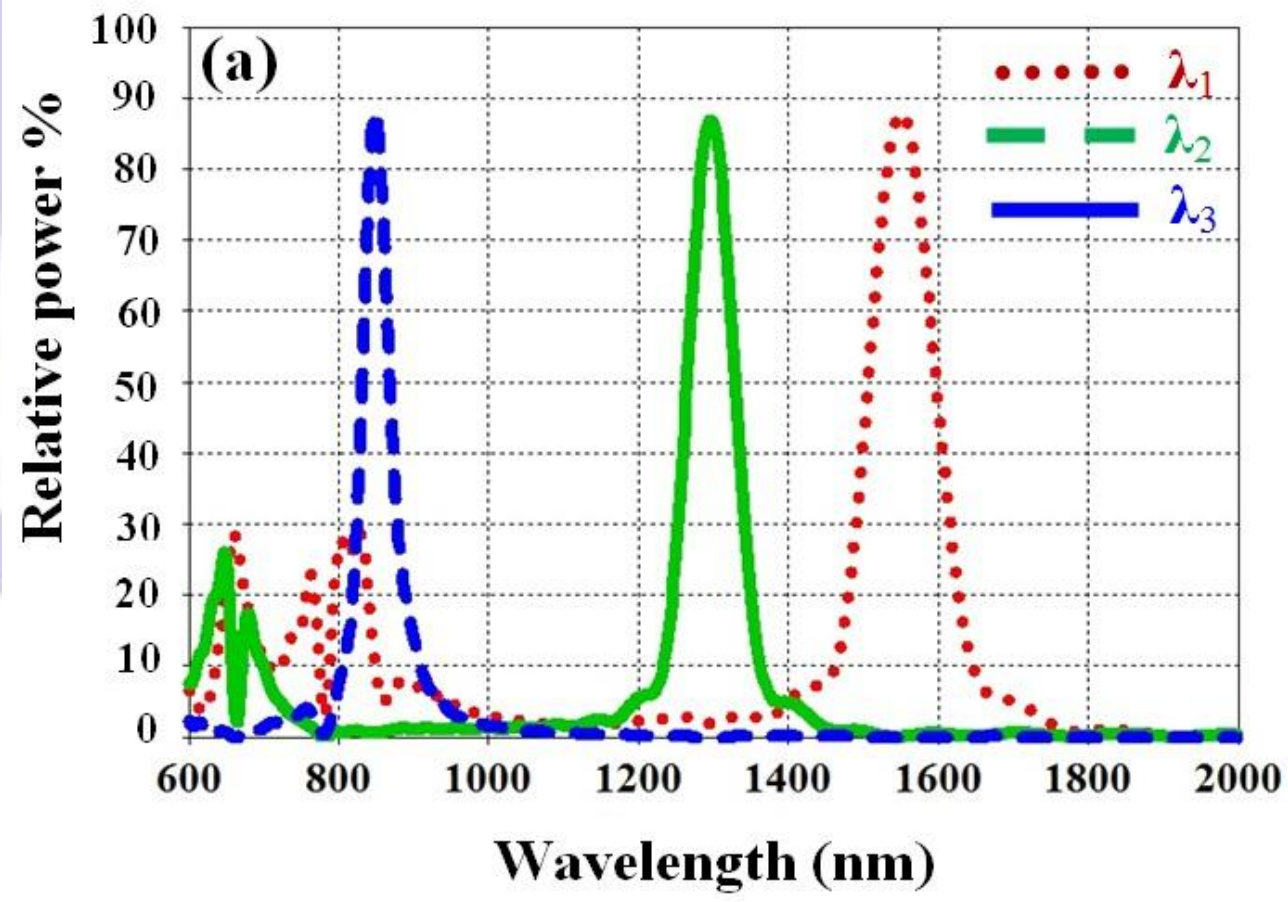

Fig. 18: (a) Power spectra associated with the demultiplexer drop ports. (b, c, and d) field distribution of $\left|\mathrm{H}_{z}\right|$ with incident wavelength 1550,1300 , and $850 \mathrm{~nm}$ respectively. 


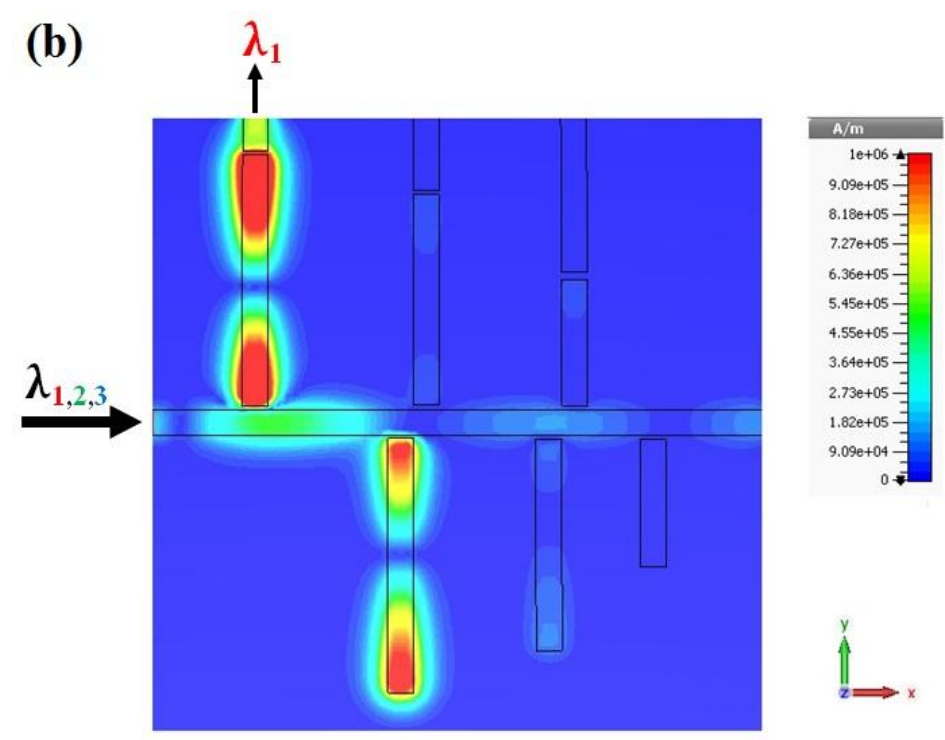

(c)
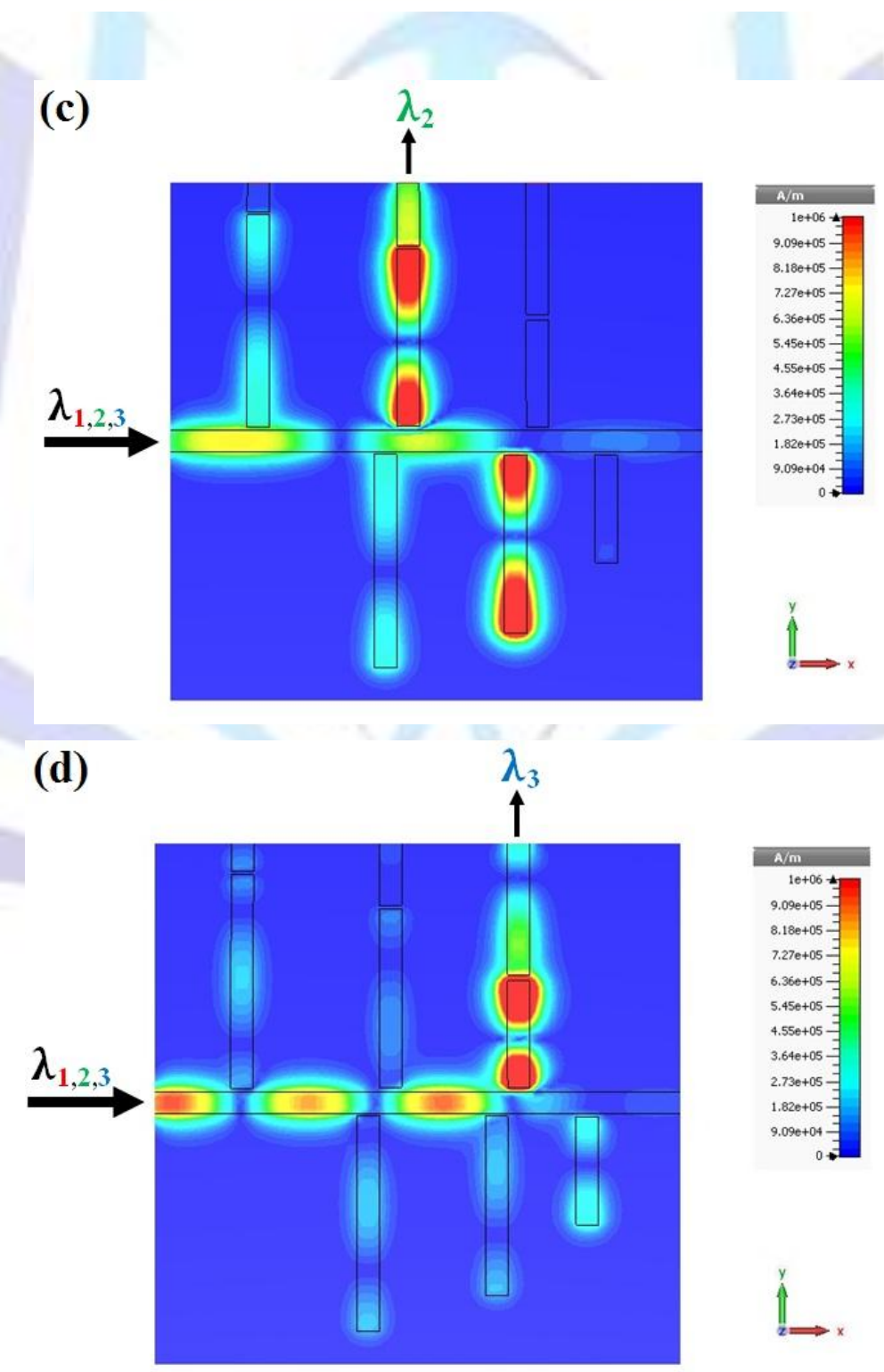

Fig. 18: (Continued). 


\section{Acknowledgments}

The authors gratefully acknowledge support from College of Engineering, Alnahrain University, Baghdad, Iraq. Part of this work will be presented at the First Postgraduate Engineering Conference to be hold at this University On November 2013.

\section{CONCLUSIONS}

In this paper, the design of 1550,1300 , and $850 \mathrm{~nm}$ silver-air-silver demultiplexer for optical communication system has been investigated. The designed structure consists of a drop nanocavity coupled with a feedback nanocavity. A design methodology has been represented which based on tuning the geometric parameters of each nanocavity separately. A drop transmission coefficient as high at $88 \%$ is obtained at 1550,1300 , and $850 \mathrm{~nm}$ for the three-channe demultiplexer, which is higher than that reported in the relevant published work.

\section{REFERENCES}

[1] A. Rahimzadegan, S. Hosseini, and K. Qaderi "Improved plasmonic demultiplexer based on tapered and rectangular slot MIM waveguide", Journal of World Academy of Science, Engineering and Technology, Vol. 77, No. 91, PP. 550-554, May 2013.

[2] M. Tiana, P. Lu, L. Chen, D. Liu and N. Peyghambarian, "Plasmonic bragg reflectors based on metal-embedded MIM structure", Optics Communications, Vol. 285, No. 24, pp. 5122-5127, November 2012.

[3] N. Nozhat and N. Granpayeh, "Analysis of the plasmonic power splitter and mux/demux suitable for photonic integrated circuits", Optics Communications Vol. 284, No. 13, pp. 3449-3455, June 2011.

[4] F. Hu, H. Yi, and Z. Zhou, "Wavelength demultiplexing structure based on arrayed plasmonic slot cavities", Optics Letters, Vol. 36, No. 8, PP. 1500-1502, April 2011.

[5] M. Farahani, N. Granpayeh, and M. Rezvani, "Improved plasmonic splitters and demultiplexers", Journal of Photonics and Nanostructures-Fundamentals and Applications, Vol. 11, No. 2, PP. 157-165, May 2013.

[6] H. Lu, X. Liu, D. Mao, L. Wang, and Y. Gong, "Tunable band-pass plasmonic waveguide filters with nanodisk resonators", Optics Express, Vol. 18, No. 17, PP. 17922-17927, August 2010.

[7] F. Hu, H. Yi, and Z. Zhou, "Band-pass plasmonic slot filter with band selection and spectrally splitting capabilities", Optics Express, Vol. 19, No. 6, PP. 4848-4855, March 2011.

[8] ] J. Zhu, X. Huang, and X. Mei, "Improved models for plasmonic waveguide splitters and demultiplexers at the telecommunication wavelengths", IEEE Transactions on Nanotechnology, Vol. 10, No. 5, PP. 1166-1171, September 2011.

[9] H. Lu, X. Liu, Y. Gong, D. Mao, and L. Wang, "Enhancement of transmission efficiency of nanoplasmonic wavelength demultiplexer based on channel drop filters and reflection nanocavities", Optics Express, Vol. 19, No. 14, PP.1288512890, July 2011.

[10] M. Bahadori, A. Eshaghian, H. Hodaei, M. Rezaei, and K. Mehrany, "Analysis and design of optical demultiplexer based on arrayed plasmonic slot cavities: transmission line model", IEEE Photonics Technology Letters, Vol. 25, PP. 784786, 2013.

[11] H. Ren, C. Jiang, W. Hu, M. Gao, and J. Wang, "Photonic crystal channel drop filter with a wavelength-selective reflection micro-cavity", Optics Express, Vol. 14, No. 6, PP. 2446-2458, March 2006.

[12] S. A. Maier, "Plasmonics: Fundamentals and Applications", Springer Science and Business Media LLC, United Kingdom, 2007.

[13] H. T. Baltar, K. Drozdowicz- Tomsia, and E. M Goldys, "Propagating surface plasmons and dispersion relations for nanoscale multilayer metallic-dielectric films', ch. 6, in K. Y. Kim (ed,),"Plasmonics - Principles and Applications", InTech, Croatia, 2012.

[14] D. Sarid and W. Challener, "Modren introduction to surface plasmons", Cambridge University Press, United Kingdoom, 2010.

[15] F. Moharrami and M. Abrishamian, "Plasmonic multi-channel filters with separately tunable pass-bands", Journal of Optics, Vol. 15, No. 7, pp. 075001-075006, July 2013.

[16] Y. Guo, L. Yan, W. Pan, B.Luo, K. Wen, Z. Guo, H. Li, and X. Luo, "A plasmonic splitter based on slot cavity", Optics Express, Vol. 19, No. 15, PP. 13831-13838, July 2011

[17] C. Zhao, Y. Li, W. Lu-Lu, Z. Yu-Fang, D. Gao-Yan, and X. Jing-Hua, "High-resolution compact plasmonic wavelength demultiplexers based on cascading square resonators", Chinese Physics Letters, Vol. 30, No. 5, PP. 054212-054215, May 2013. 


\section{APPENDIX A : $1300 \mathrm{~nm}$ DEMULTIPLEXER}

The demultiplexer is designed using the following geometric parameters $W_{1}=W_{2}=W_{t}=50 \mathrm{~nm}, L_{1}=L_{2}=406$ $\mathrm{nm}, g_{1}=7 \mathrm{~nm}, g_{2}=6 \mathrm{~nm}, g_{d}=10 \mathrm{~nm}$ and $D=236 \mathrm{~nm}$, the simulation results are presented in Figs. A1-A11.
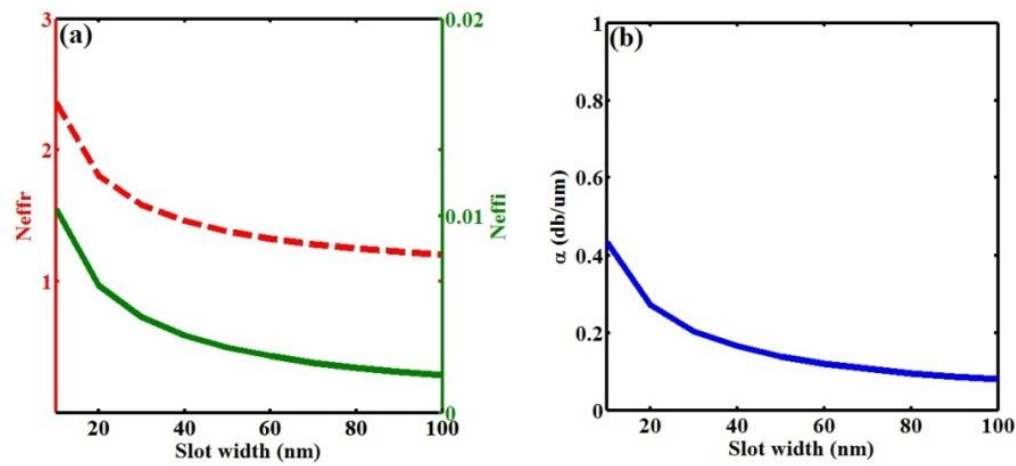

Fig. A-1: (a) Variation of the real and imaginary parts of (ERI) with air slot width at $\lambda=1300 \mathrm{~nm}$. (b) dependence of the waveguide attenuation on the width.
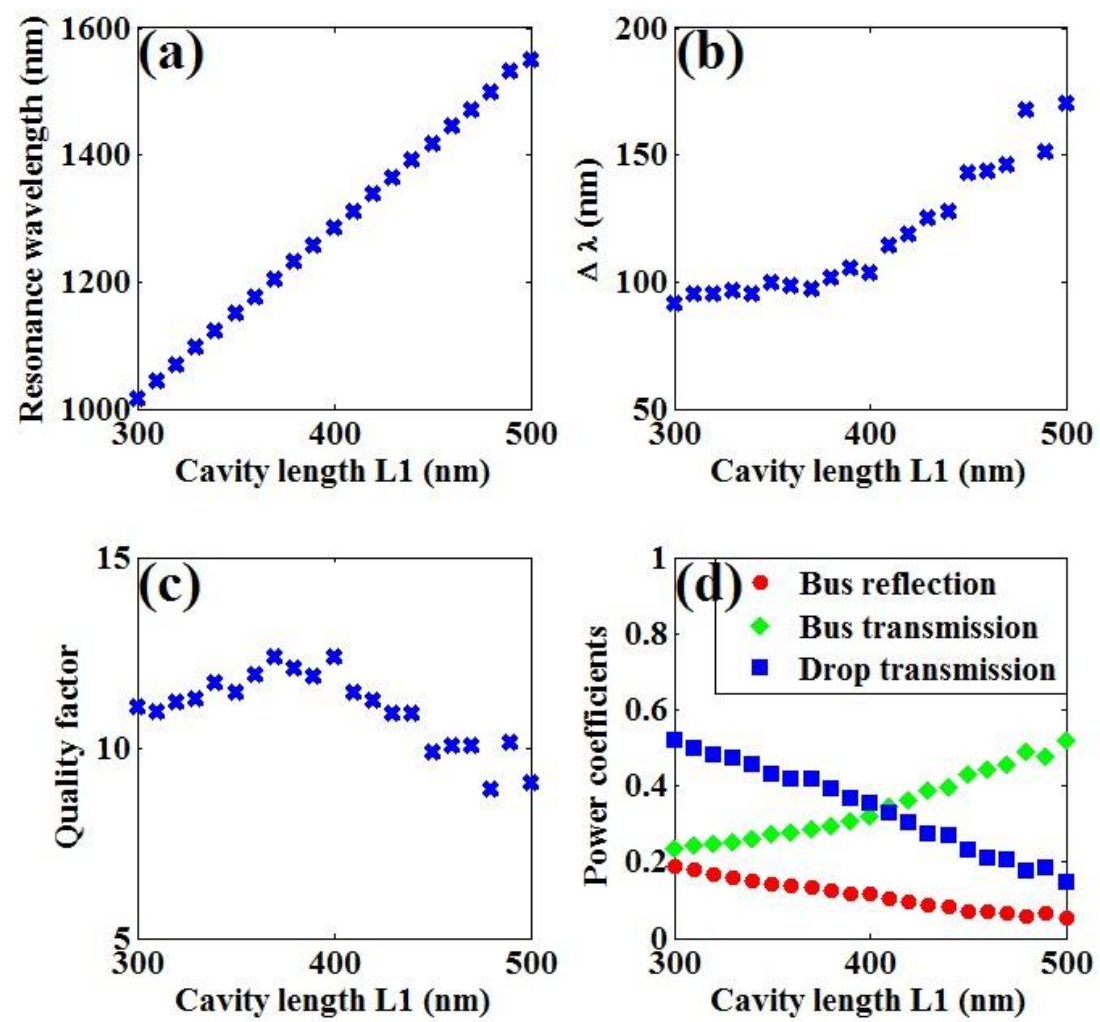

Fig. A-2: Effect of cavity length $L_{1}$ on (a) resonance wavelength, (b) spectrum bandwidth, (c) quality factor, and (d) power coefficients. 

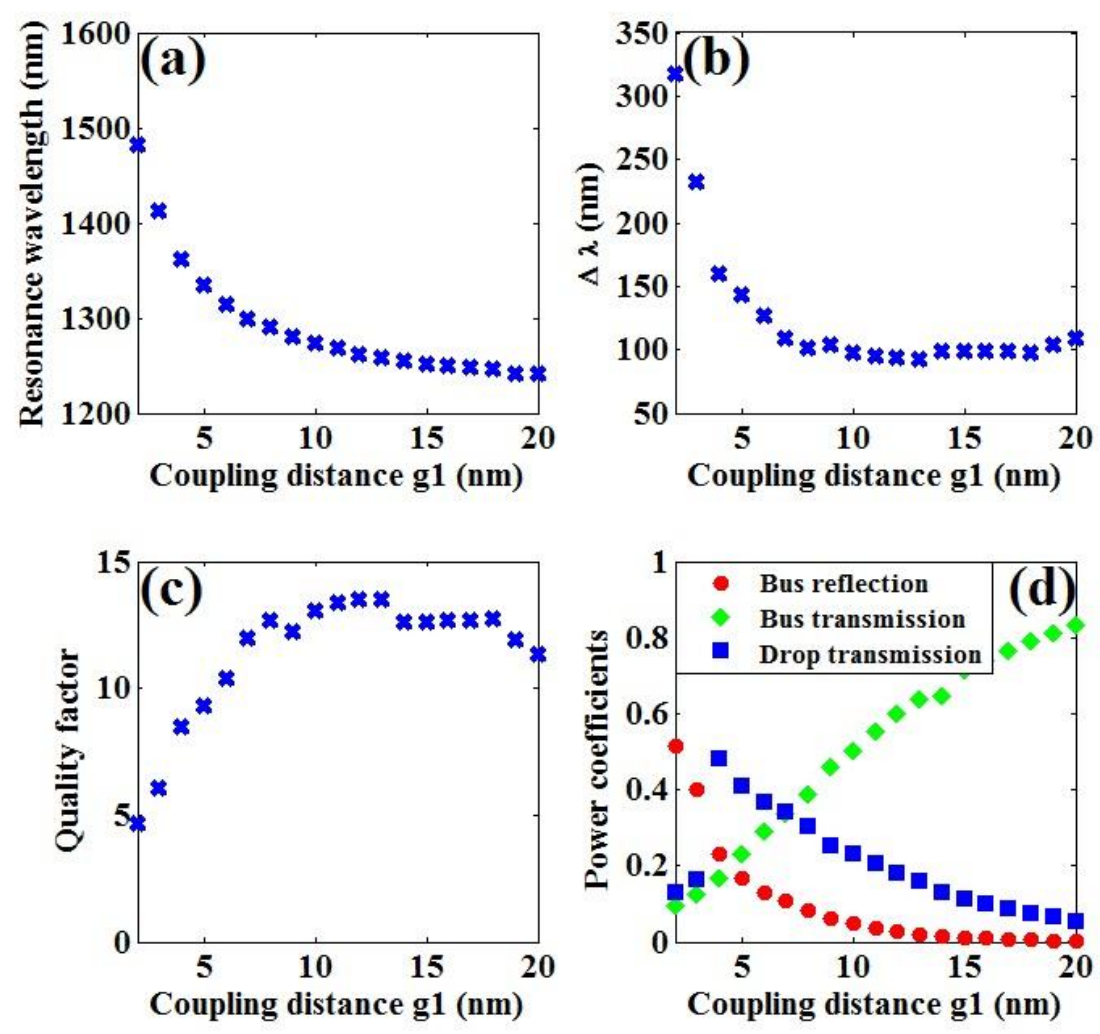

Fig. A-3: Effect of cavity length $g_{1}$ on (a) resonance wavelength, (b) spectrum bandwidth, (c) quality factor, and (d) power coefficients.
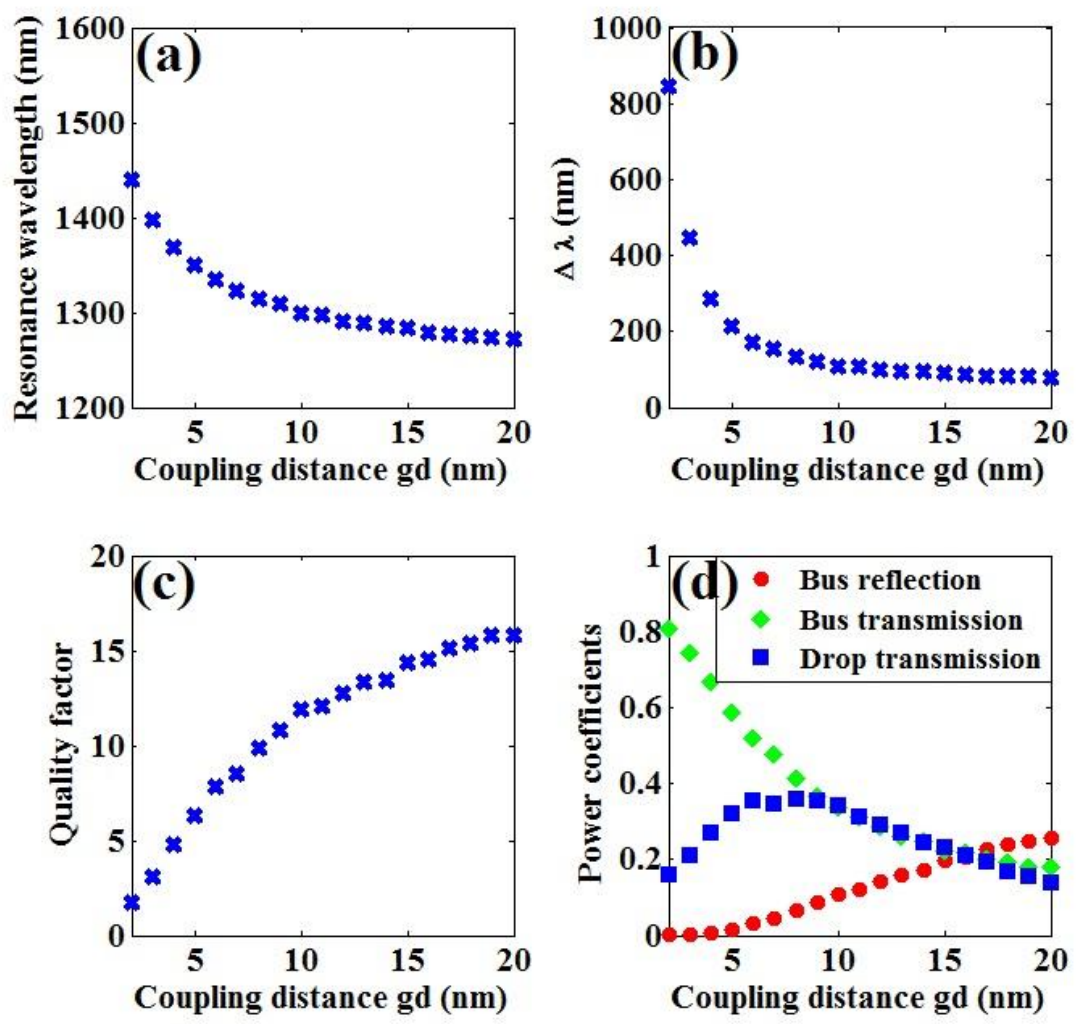

Fig. A-4: Effect of cavity length $g_{d}$ on (a) resonance wavelength, (b) spectrum bandwidth, (c) quality factor, and (d) power coefficients. 

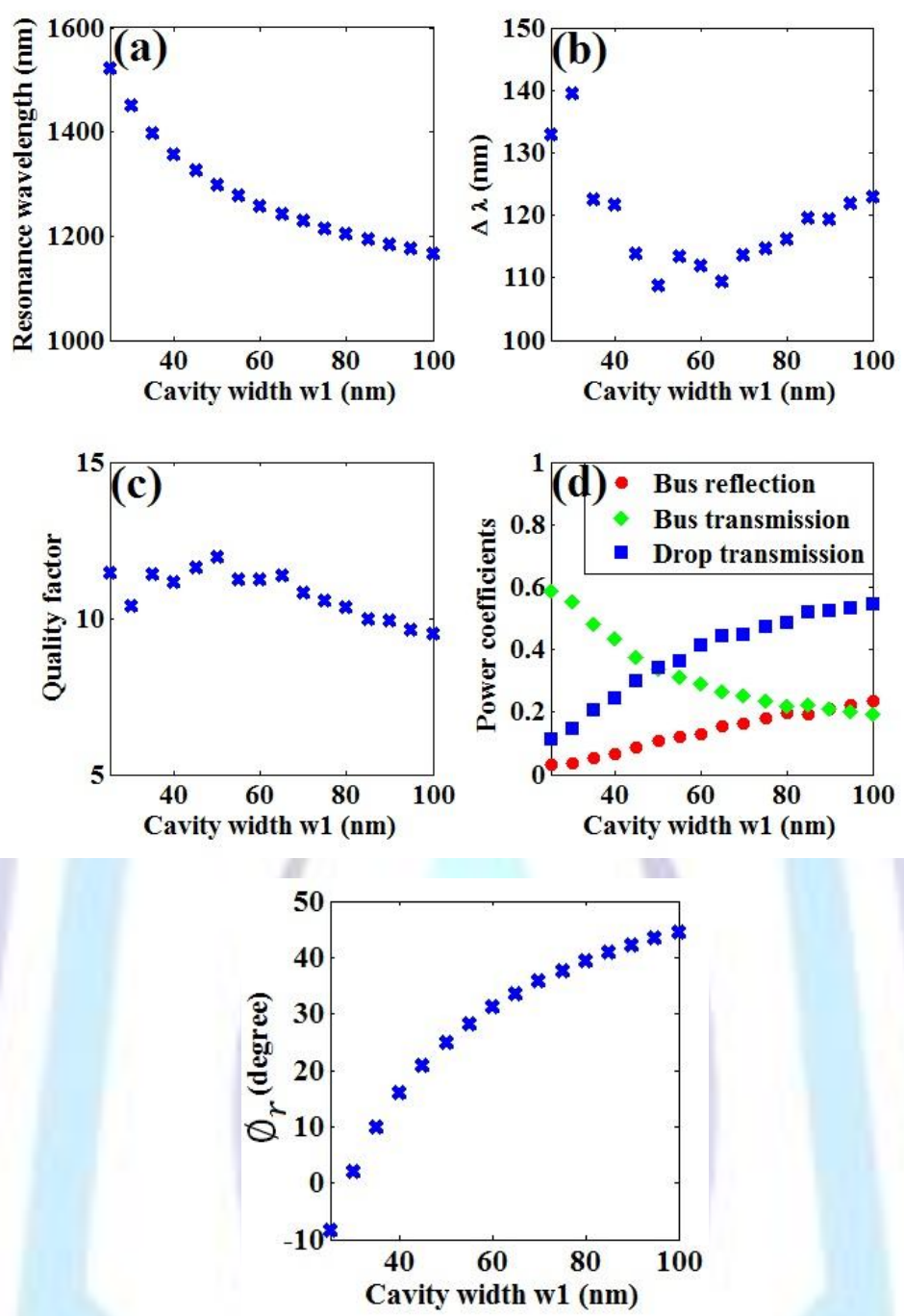

Fig. A-5: Effect of cavity length $w_{1}$ on (a) resonance wavelength, (b) spectrum bandwidth, (c) quality factor, (d) power coefficients, and (e) phase shift of SPPs reflected at one facet of the cavity.

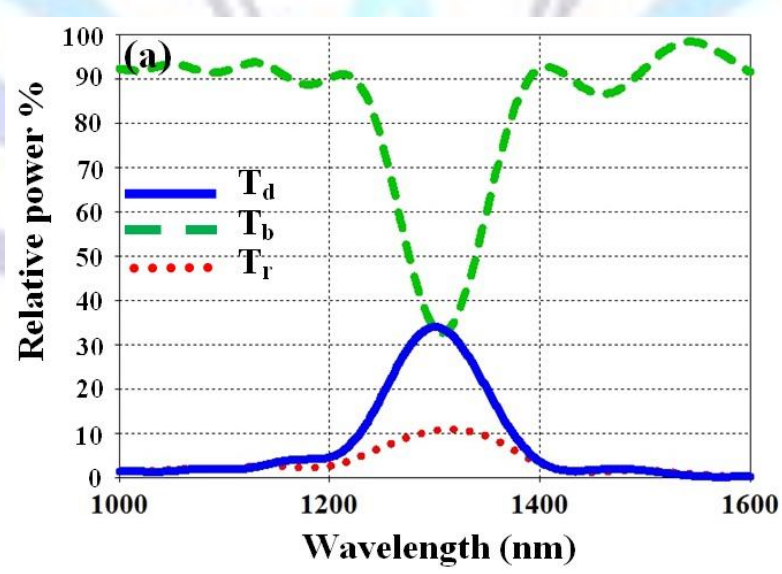




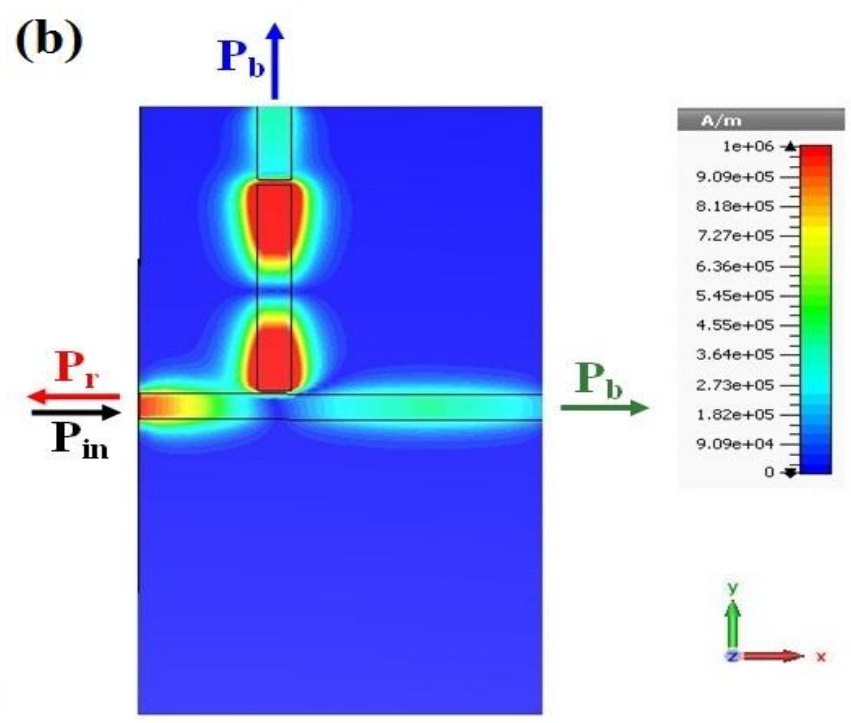

Fig. A-6: (a) Power spectra associated with the cavity 1. (b) field distribution of $\left|H_{z}\right|$ with incident wavelength of $1300 \mathrm{~nm}$.
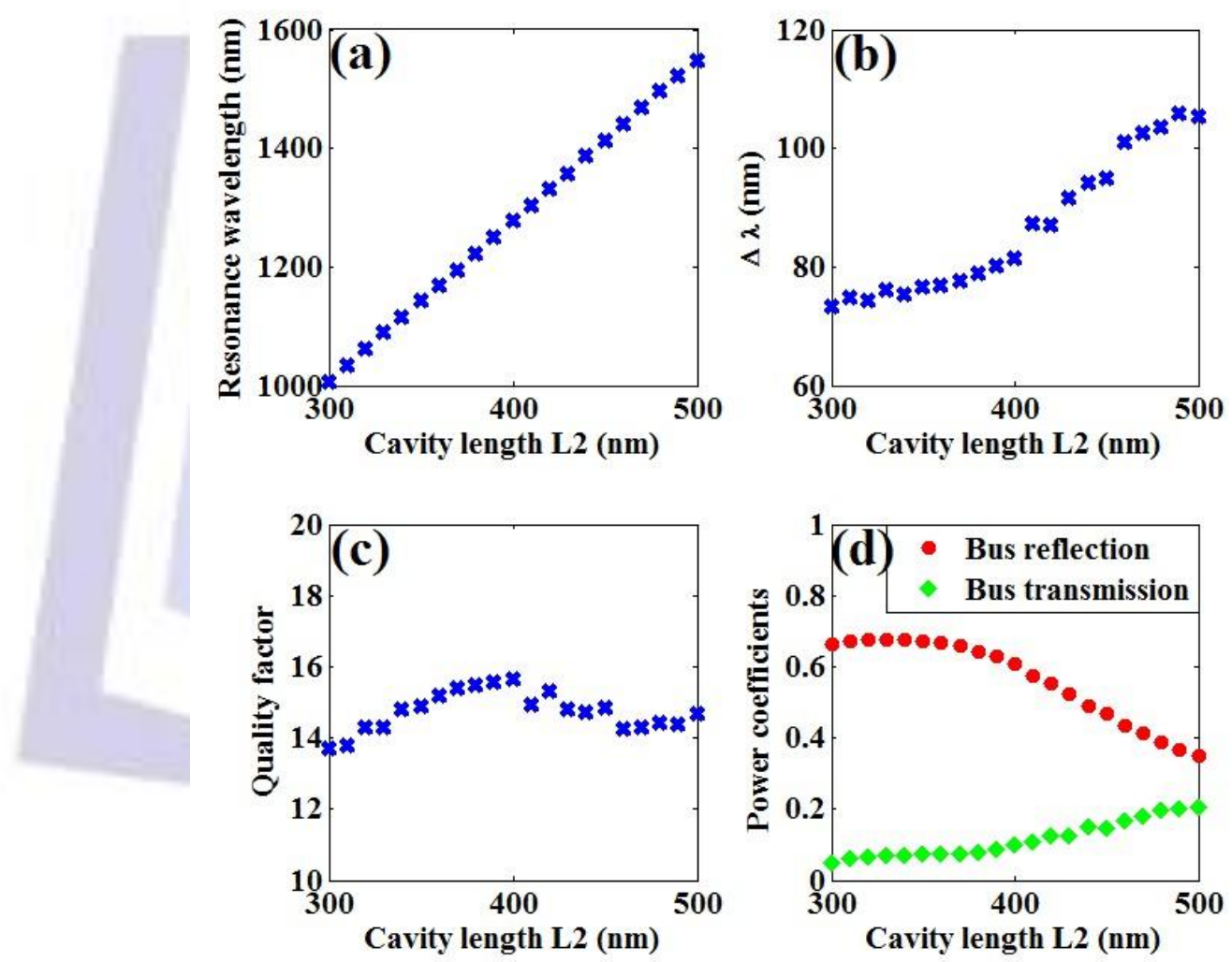

Fig. A-7: Effect of cavity length $L_{2}$ on (a) resonance wavelength, (b) spectra bandwidth, (c) quality factor, and (d) power coefficients. 

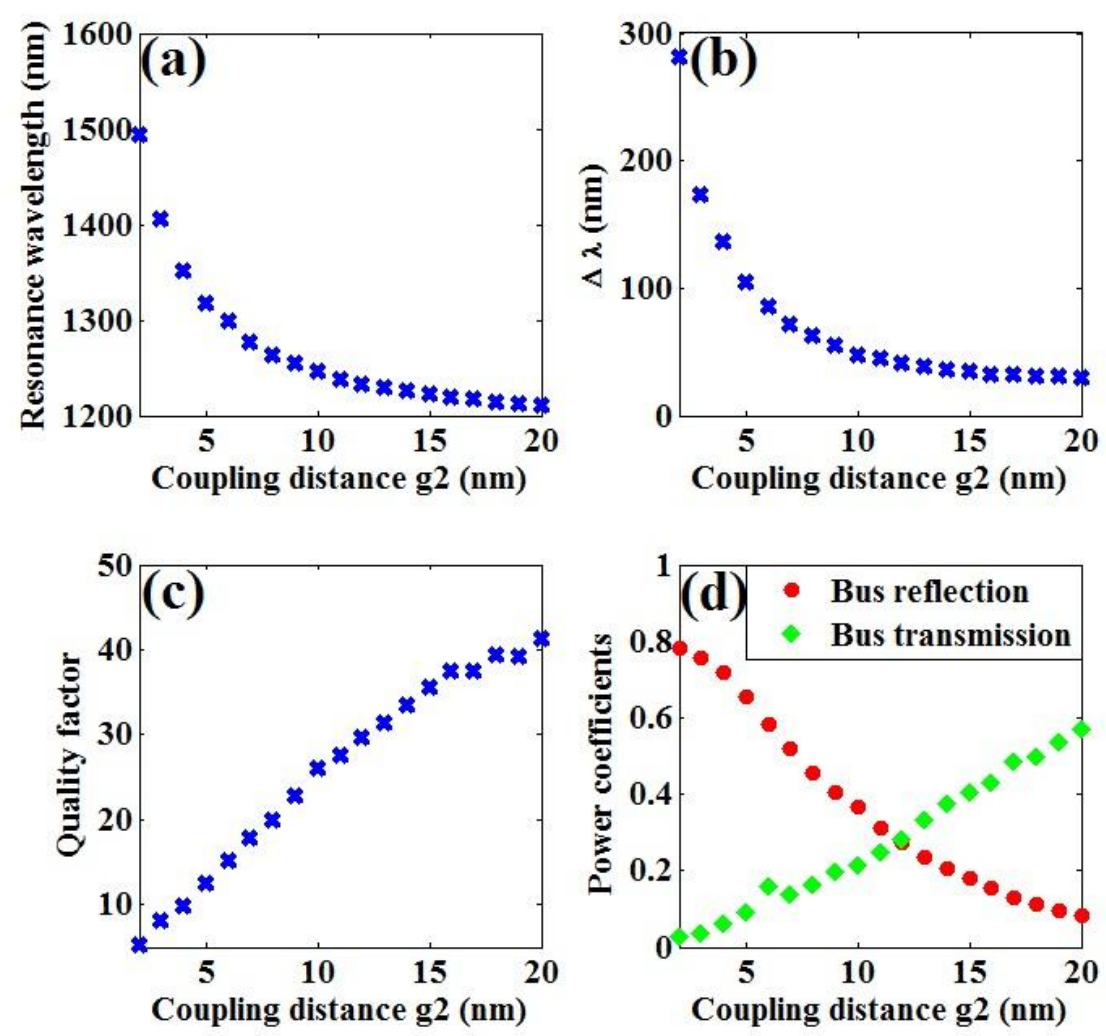

Fig. A-8: Effect of cavity length $g_{2}$ on (a) resonance wavelength, (b) spectrum bandwidth, (c) quality factor, and (d) power coefficients.
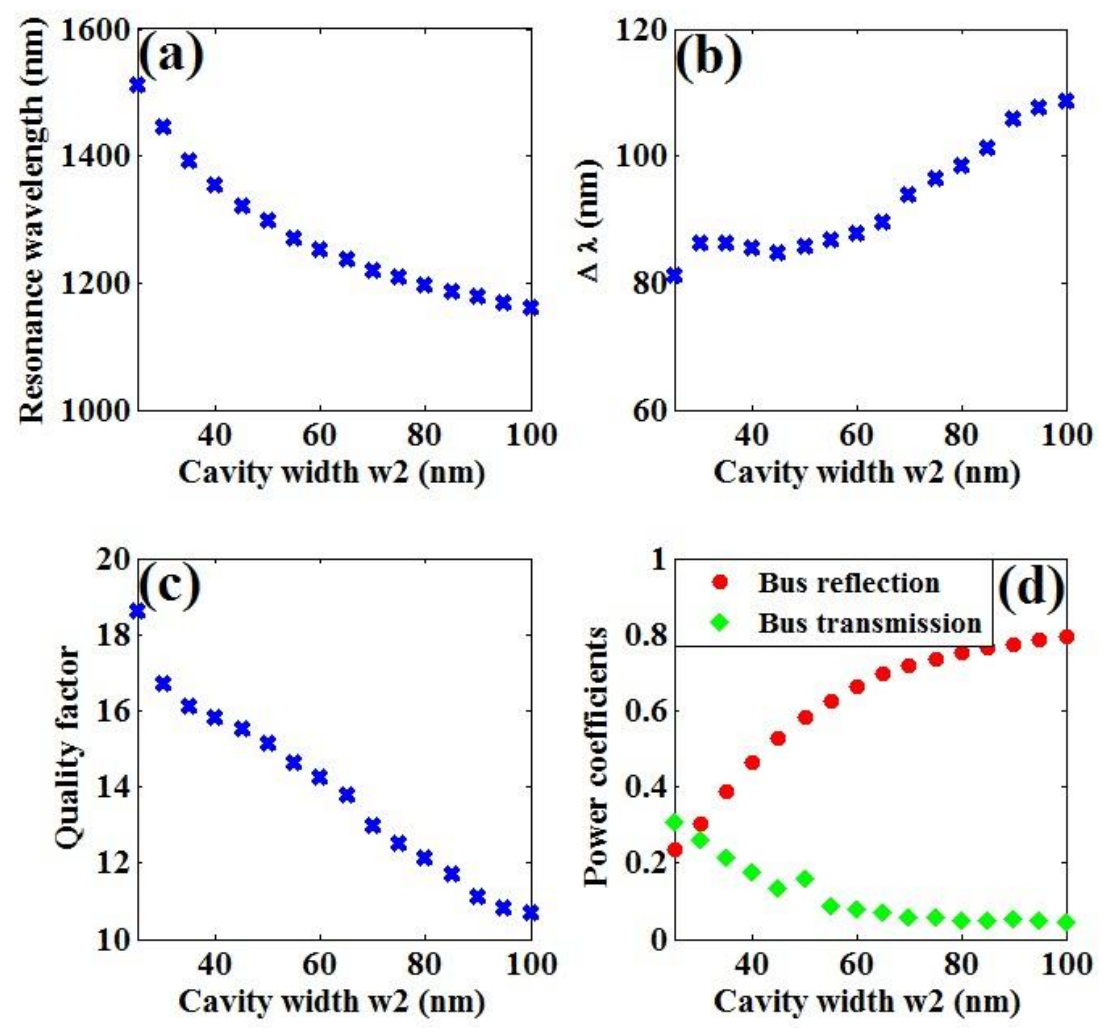


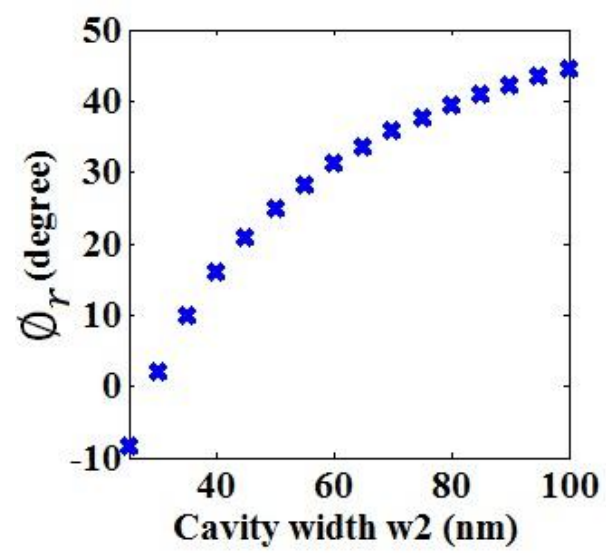

Fig. A-9: Effect of cavity length $w_{2}$ on (a) resonance wavelength, (b) spectrum bandwidth, (c) quality factor, (d) power coefficients, and (e) phase shift of SPPs reflected at one facet of the cavity.

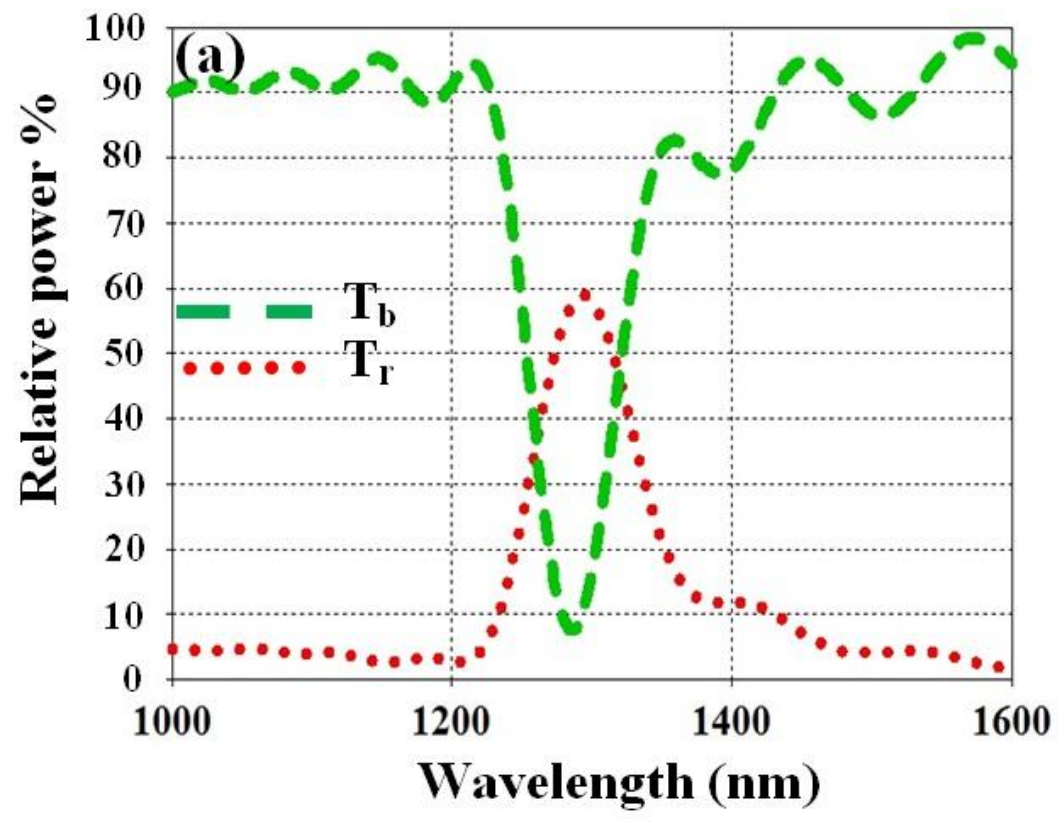

(b)

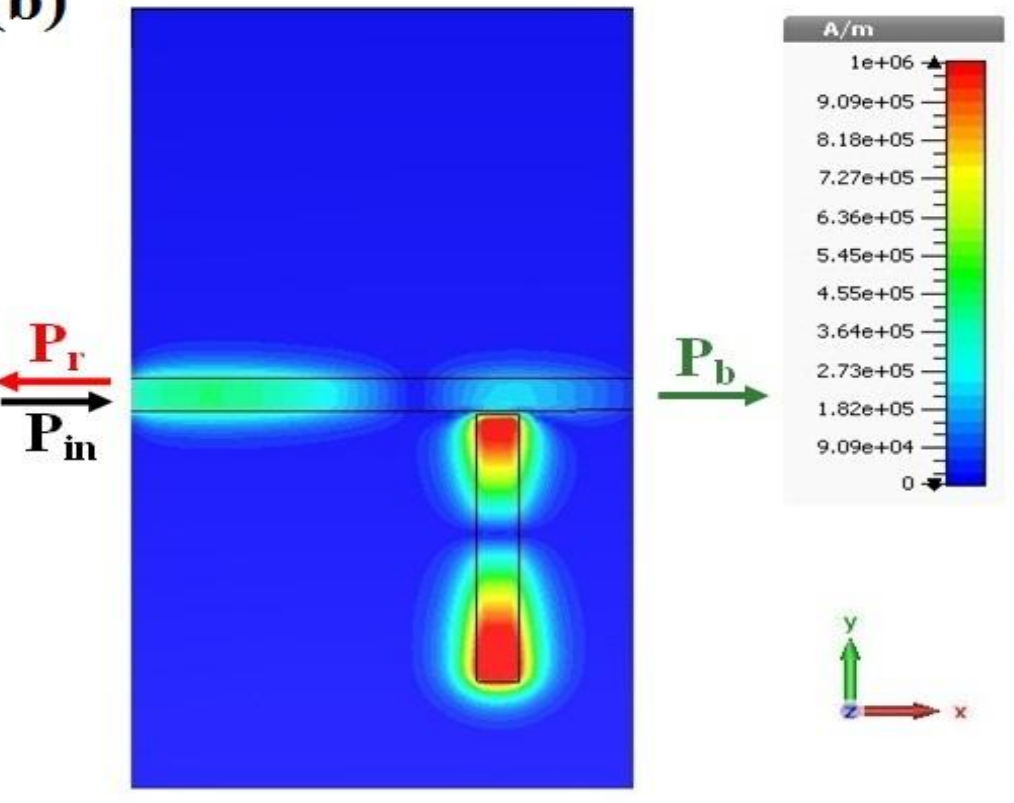


Fig. A-10: (a) Power spectra associated with the cavity 2. (b) field distribution of $\left|\mathrm{H}_{z}\right|$ with $1300 \mathrm{~nm}$-incident wavelength.

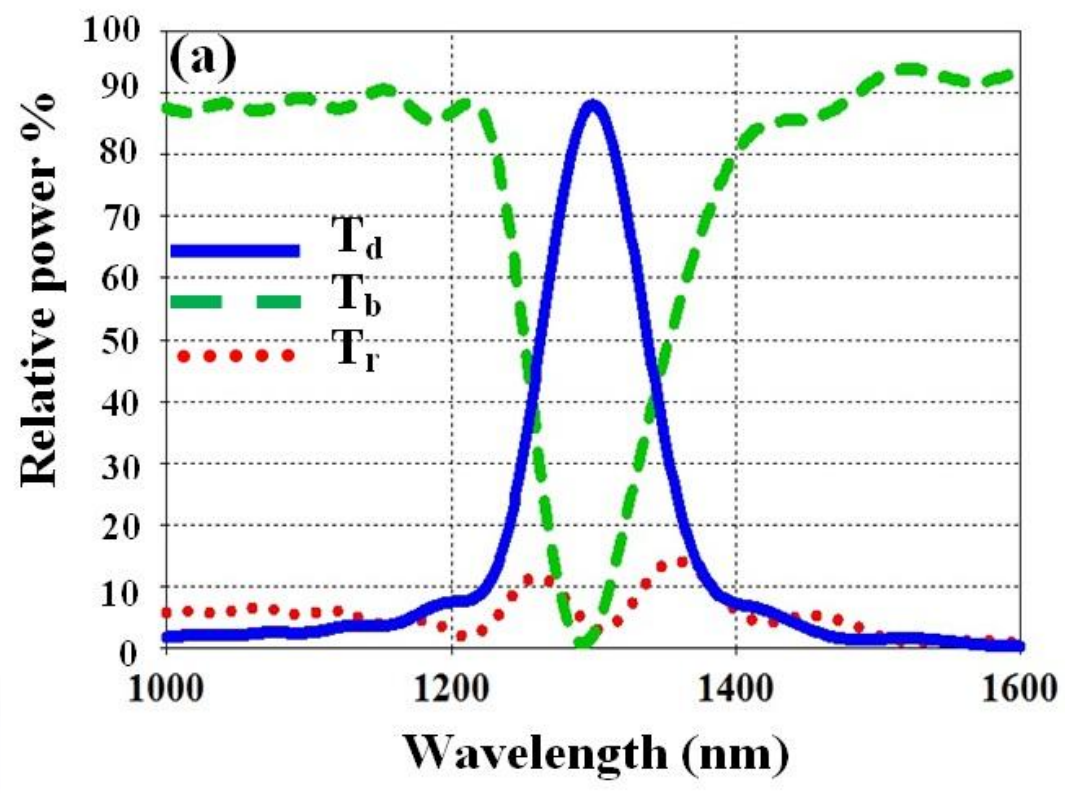

(b)
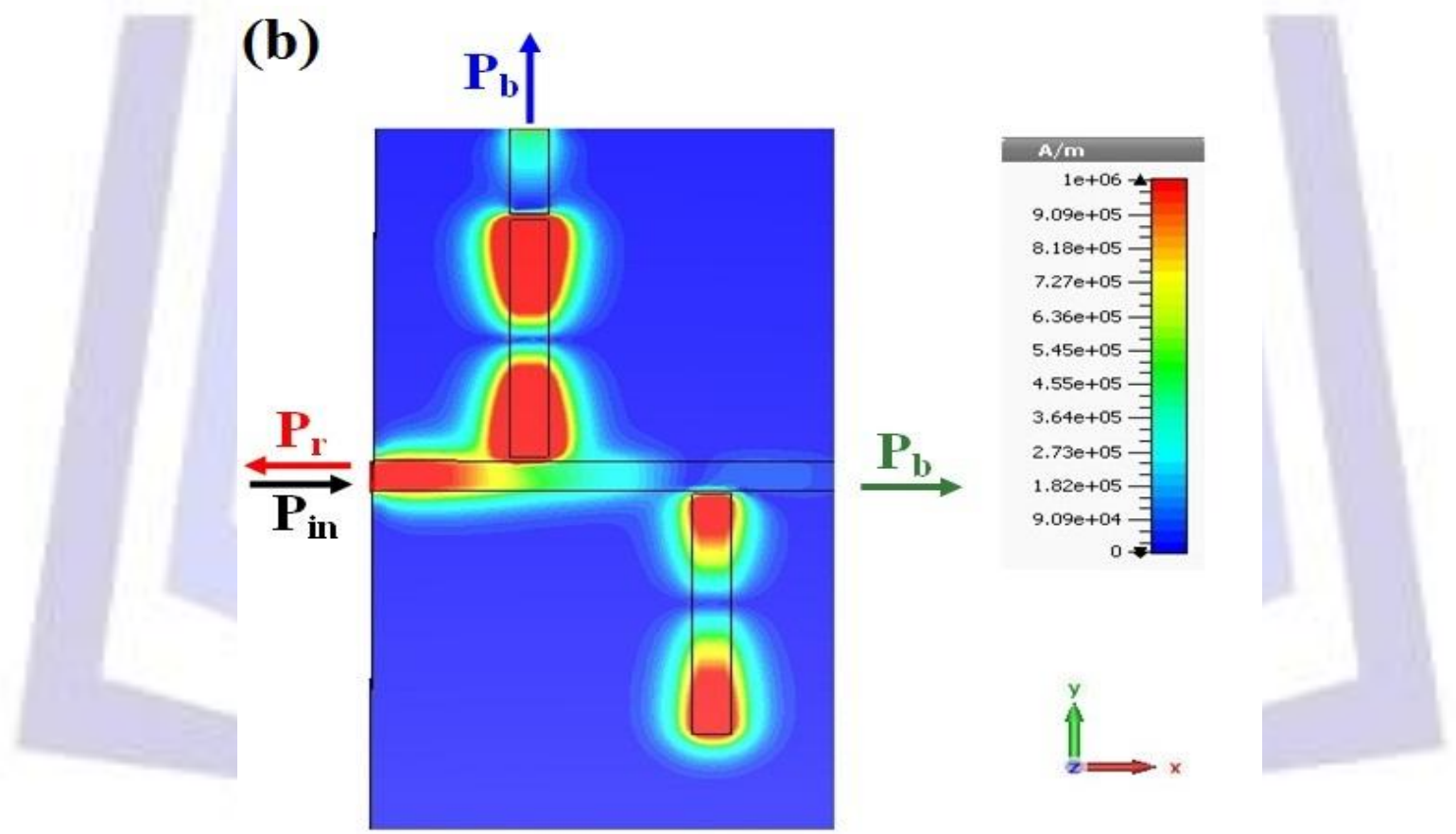

Fig. A-11: Power spectra associated with the $1300 \mathrm{~nm}$ demultiplexer. (b) field distribution of $\left|\mathrm{H}_{z}\right|$ with incident wavelength $1300 \mathrm{~nm}$. 


\section{APPENDIX B : $850 \mathrm{~nm}$ DEMULTIPLEXER}

The demultiplexer is designed using the following geometric parameters $W_{1}=W_{2}=W_{t}=50 \mathrm{~nm}, L_{1}=L_{2}=255$ $\mathrm{nm}, g_{1}=12 \mathrm{~nm}, g_{2}=8 \mathrm{~nm}, g_{d}=13 \mathrm{~nm}$, and $D=151 \mathrm{~nm}$, the simulation results are presented in Figs. B1-B11.
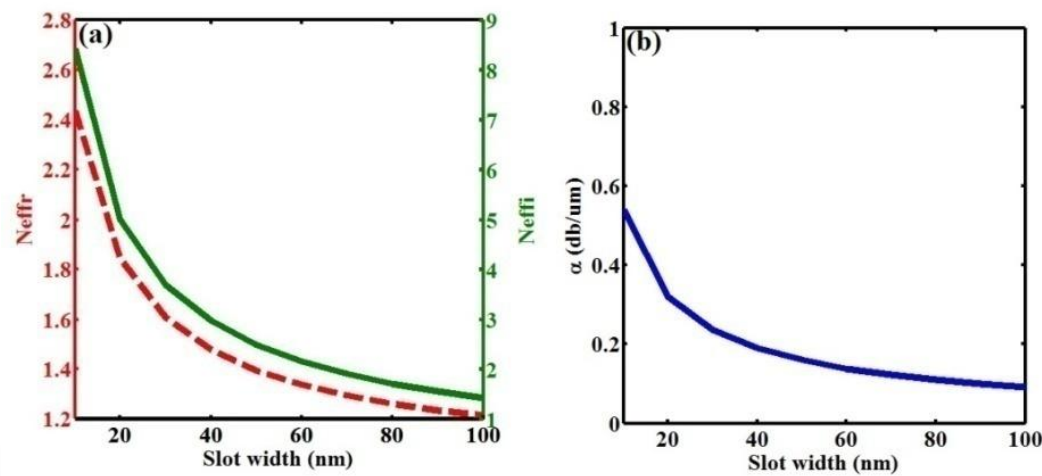

Fig. B-1: (a) Variation of the real and imaginary parts of (ERI) with air slot width at $\lambda=850 \mathrm{~nm}$. (b) dependence of the waveguide attenuation on the width.
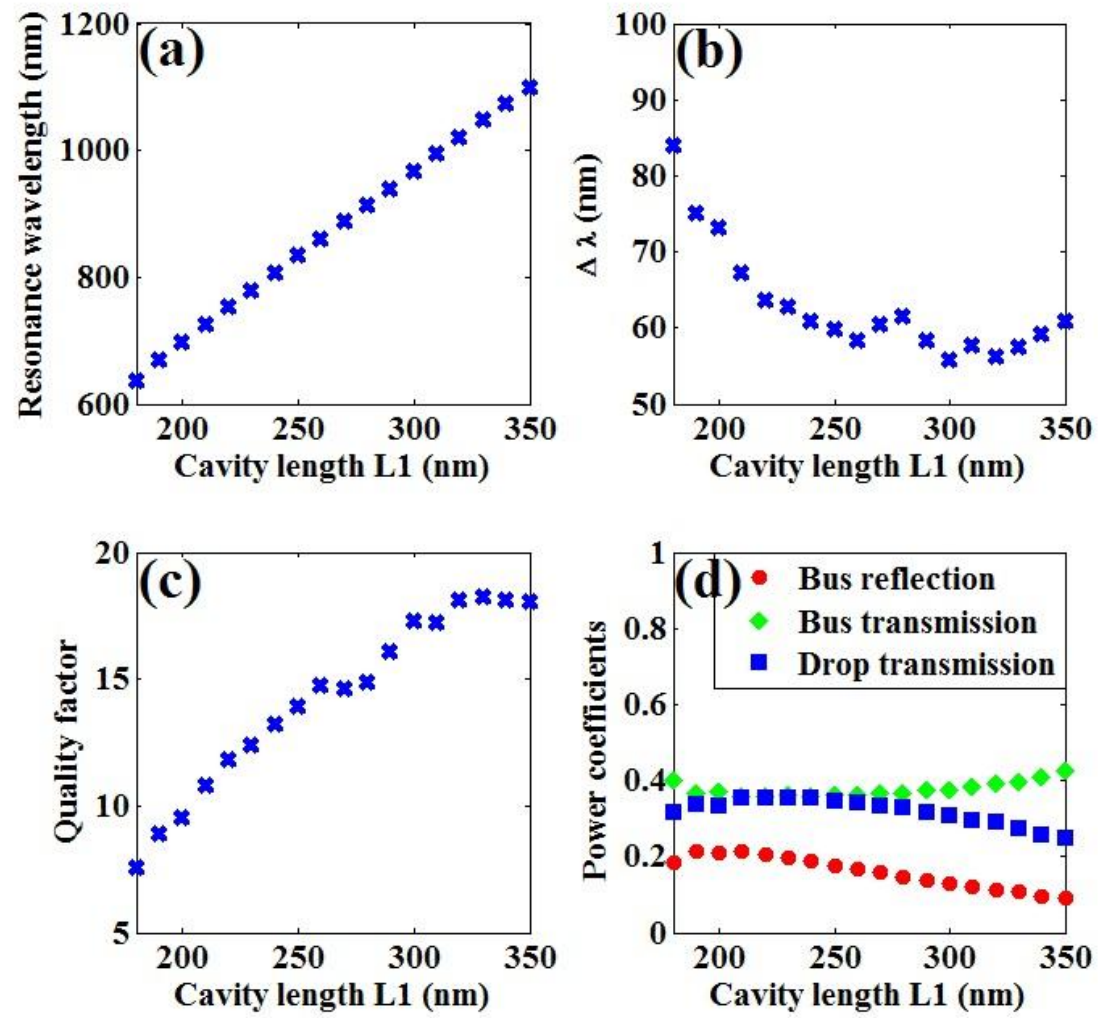

Fig. B-2: Effect of cavity length $L_{1}$ on (a) resonance wavelength, (b) spectrum bandwidth, (c) quality factor, and (d) power coefficients. 

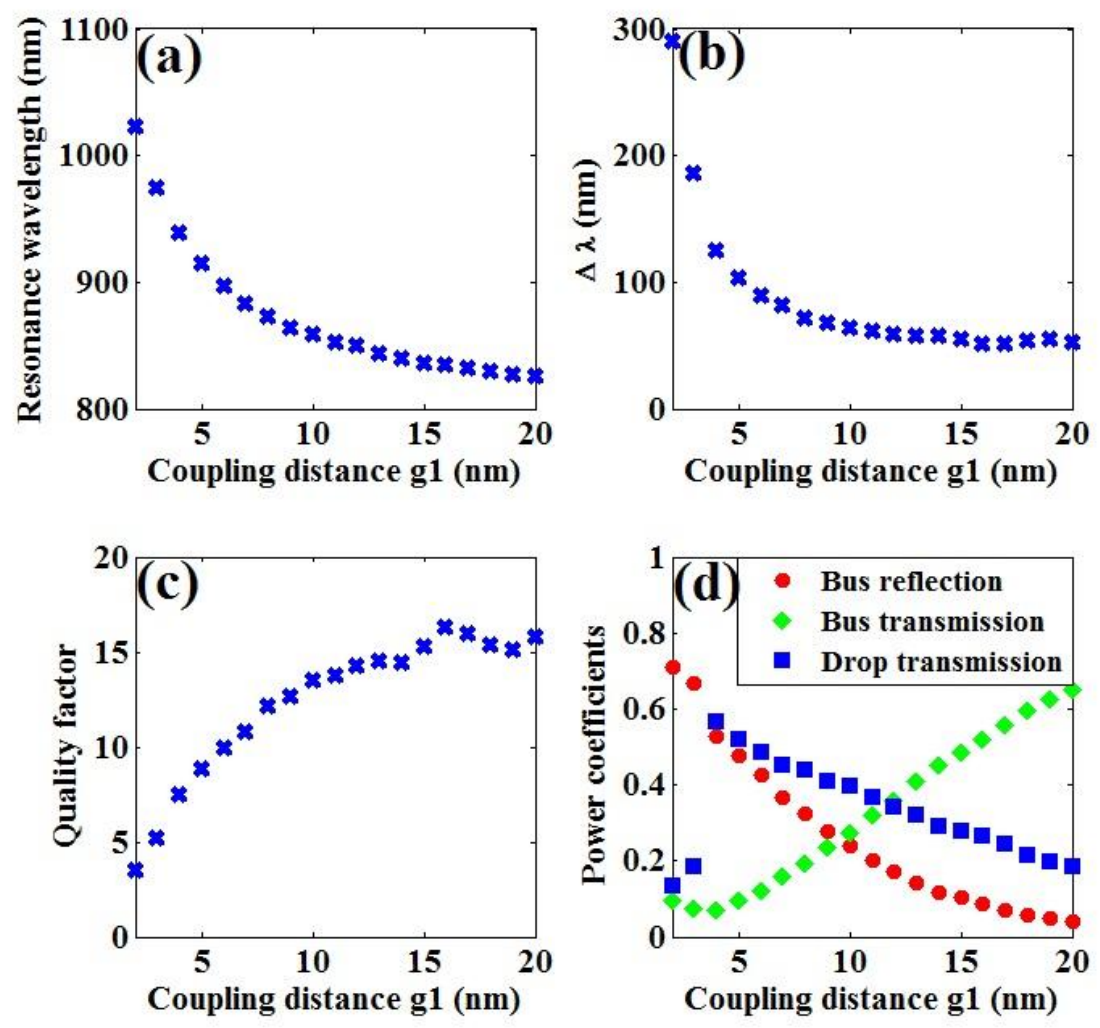

Fig. B-3: Effect of cavity length $g_{1}$ on (a) resonance wavelength, (b) spectrum bandwidth, (c) quality factor, and (d) power coefficients.
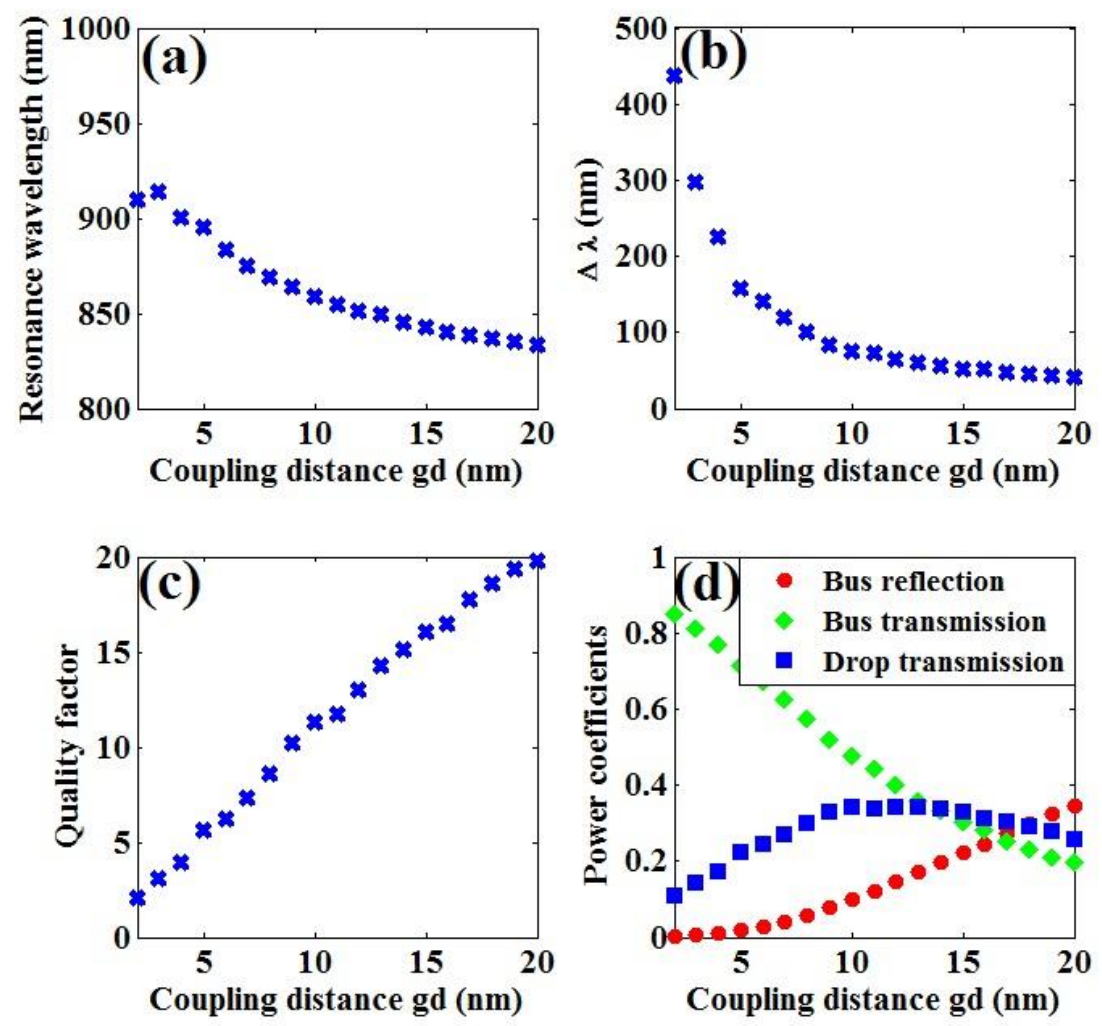

Fig. B-4: Effect of cavity length $g_{d}$ on (a) resonance wavelength, (b) spectrum bandwidth, (c) quality factor, and (d) power coefficients. 

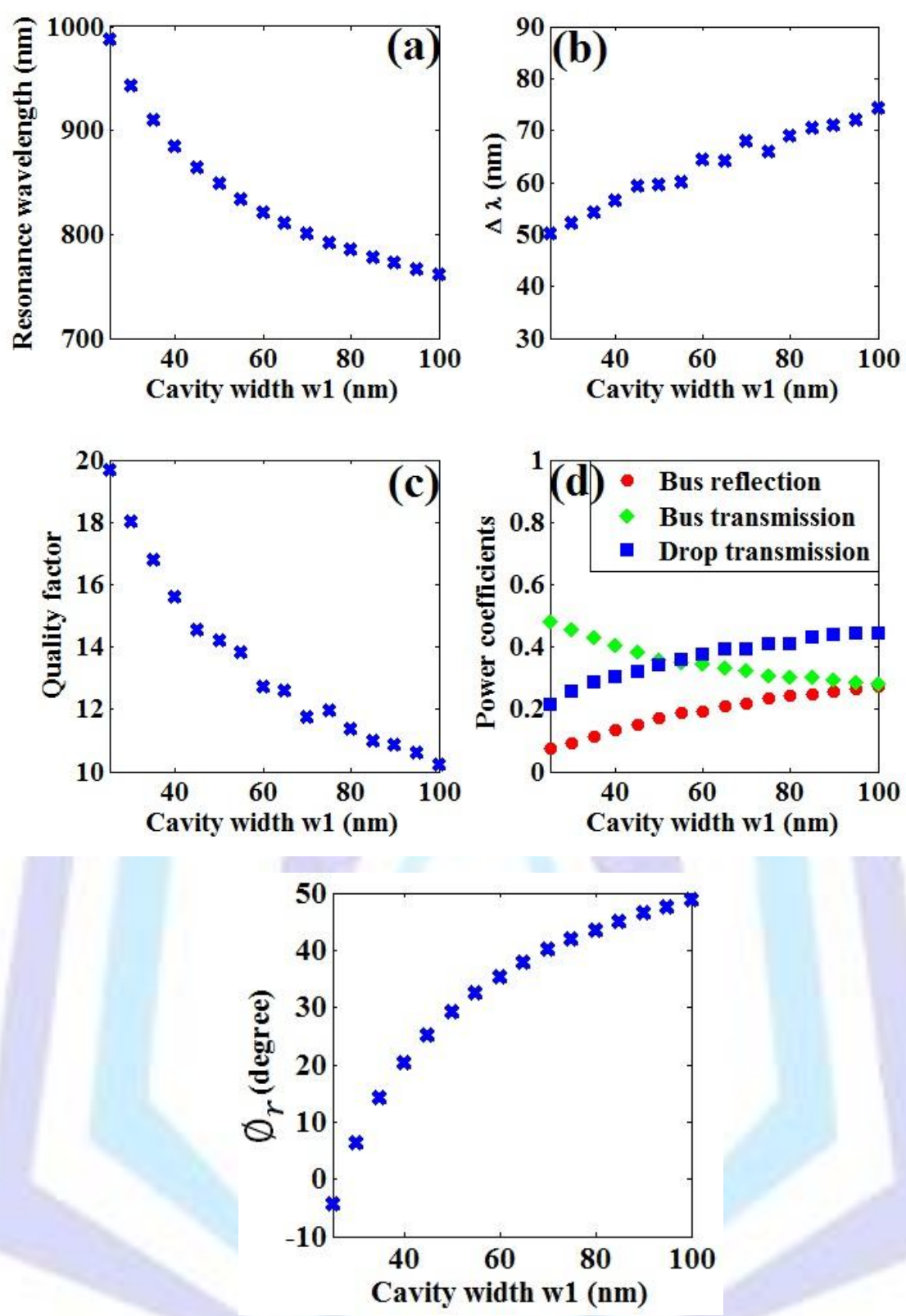

Fig. B-5: Effect of cavity length $w_{1}$ on (a) resonance wavelength, (b) spectrum bandwidth, (c) quality factor, (d) power coefficients, and (e) phase shift of SPPs reflected at one facet of the cavity. 


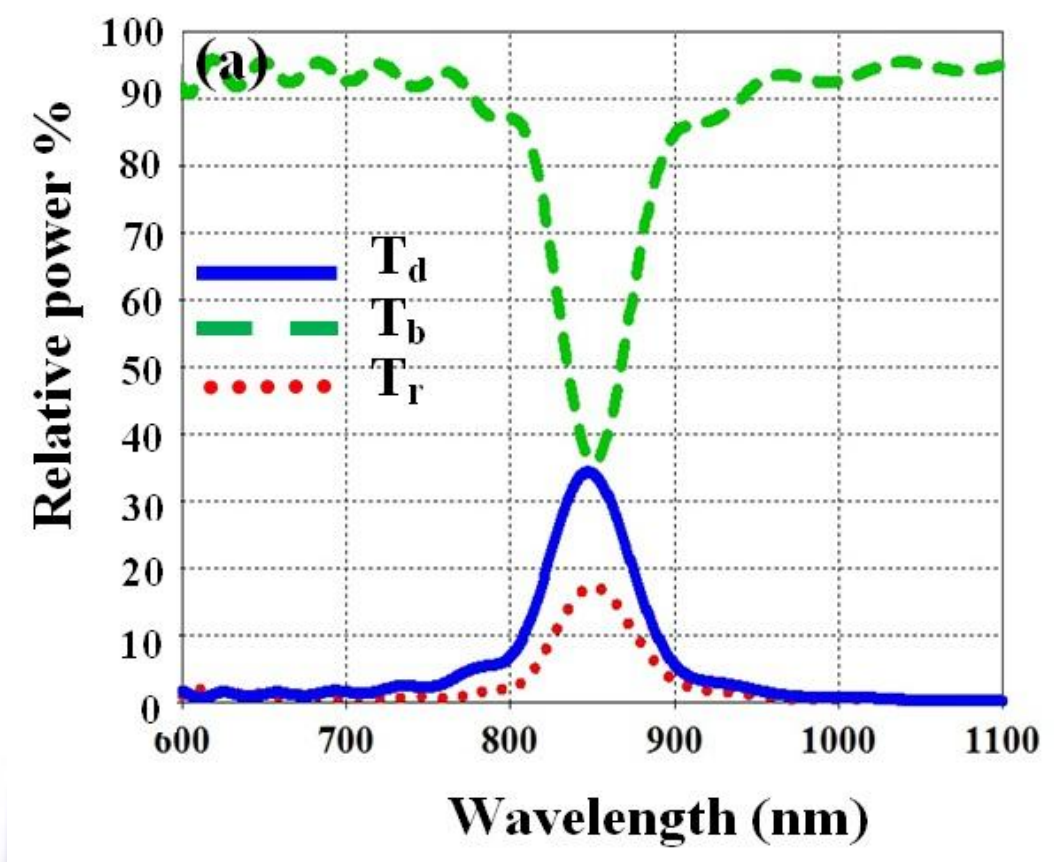

(b) $\quad \mathbf{P}_{\mathrm{b}} \uparrow$
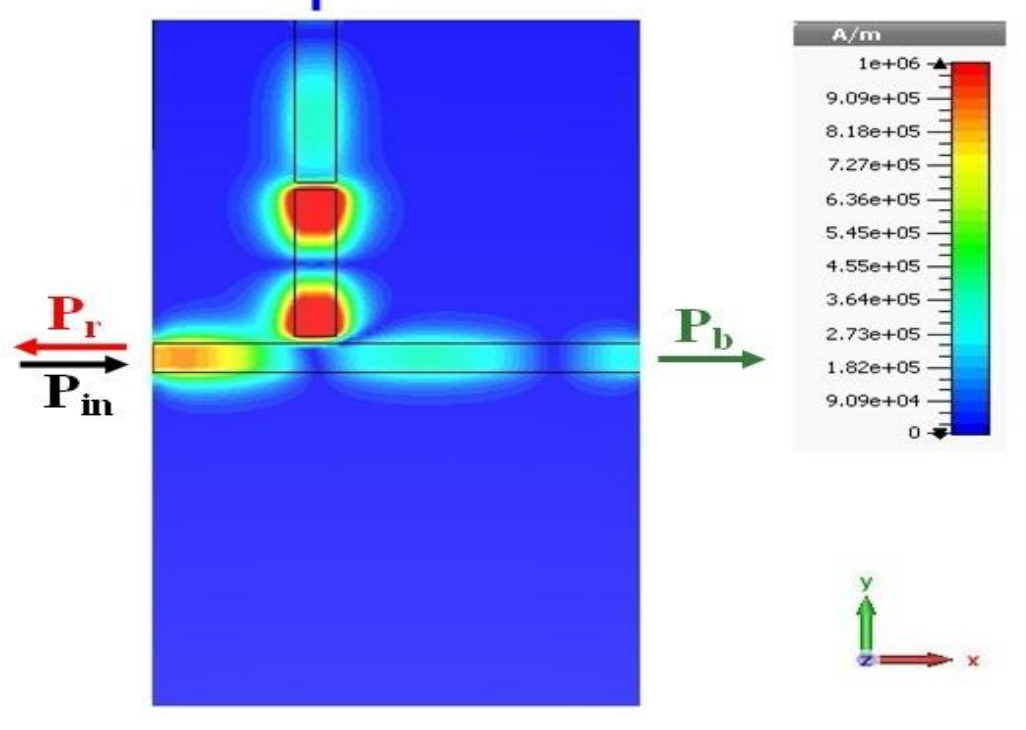

Fig. B-6: Power spectra associated with the cavity 1. (b) field distribution of $\left|\mathrm{H}_{z}\right|$ with incident wavelength of 850 $\mathrm{nm}$. 

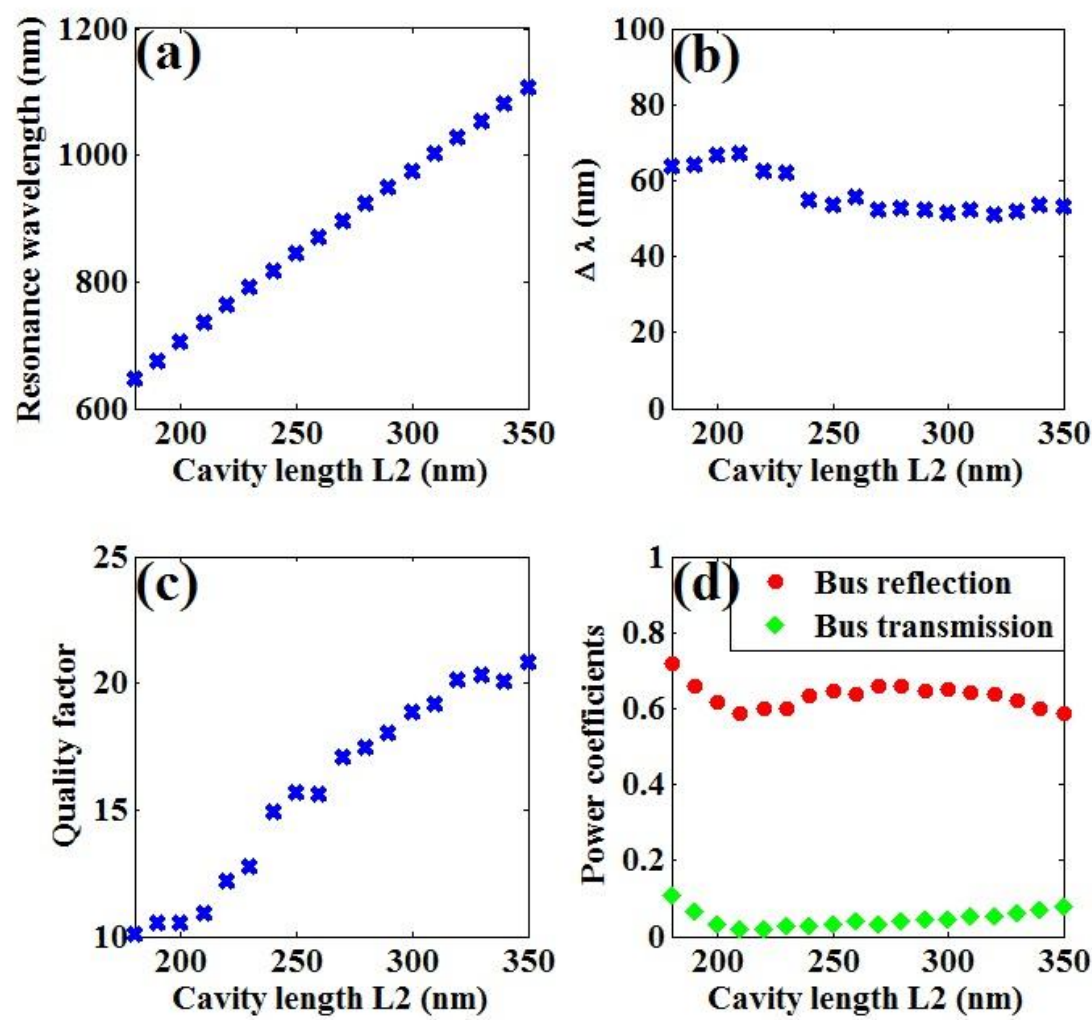

Fig. B-7: Effect of cavity length $L_{2}$ on (a) resonance wavelength, (b) spectra bandwidth, (c) quality factor, and (d) power coefficients.
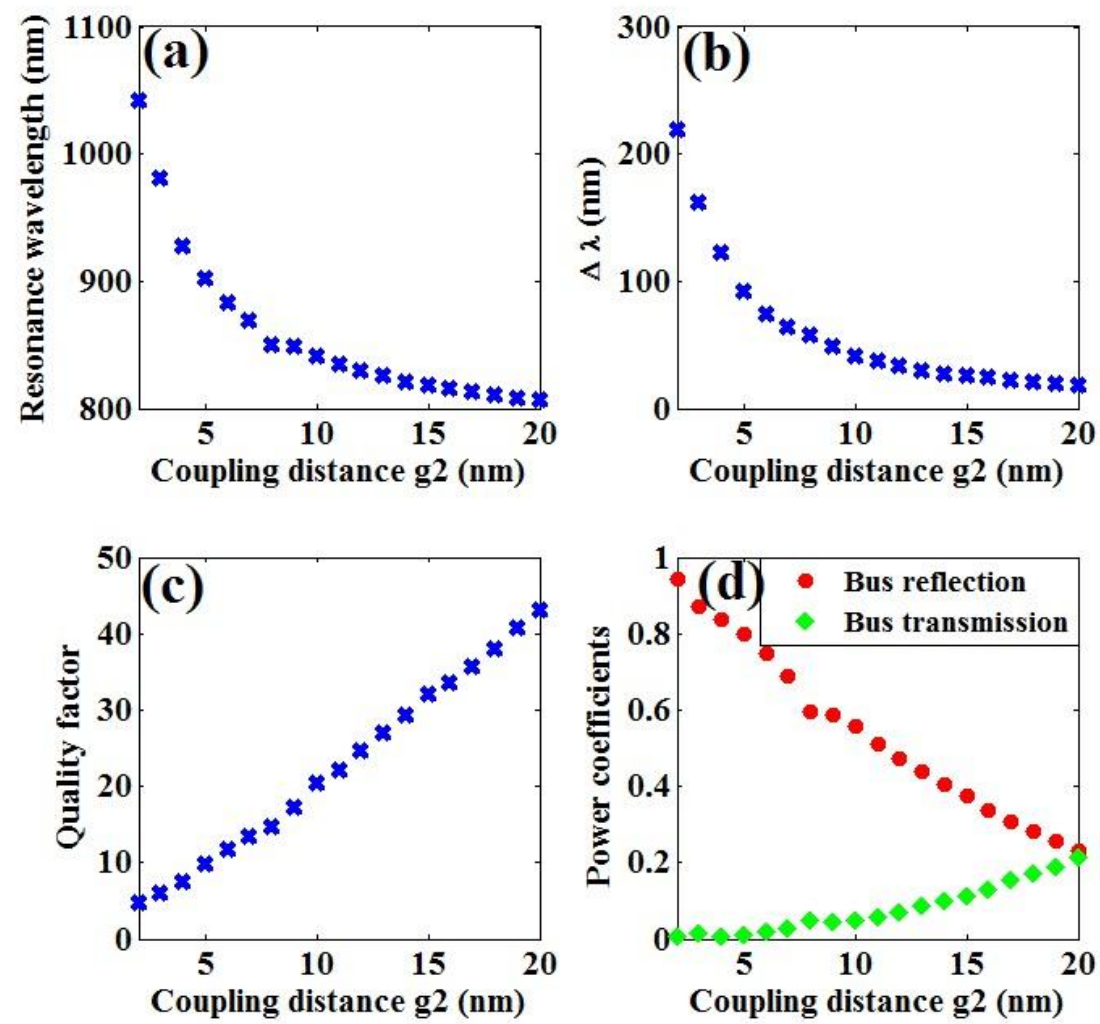

Fig. B-8: Effect of cavity length $g_{2}$ on (a) resonance wavelength, (b) spectrum bandwidth, (c) quality factor, and (d) power coefficients. 

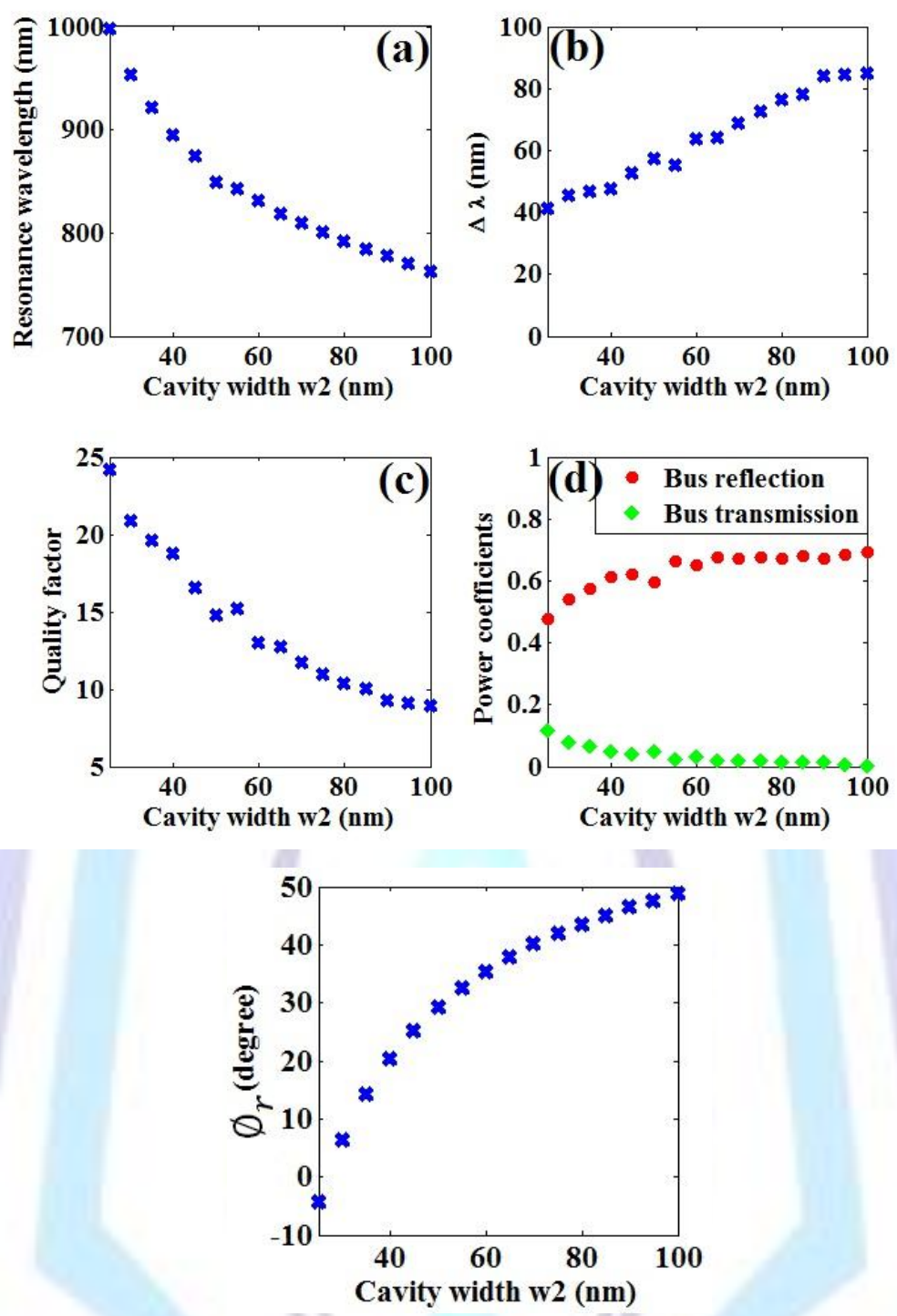

Fig. B-9: Effect of cavity length $w_{2}$ on (a) resonance wavelength, (b) spectrum bandwidth, (c) quality factor, (d) power coefficients, and (e) phase shift of SPPs reflected at one facet of the cavity.

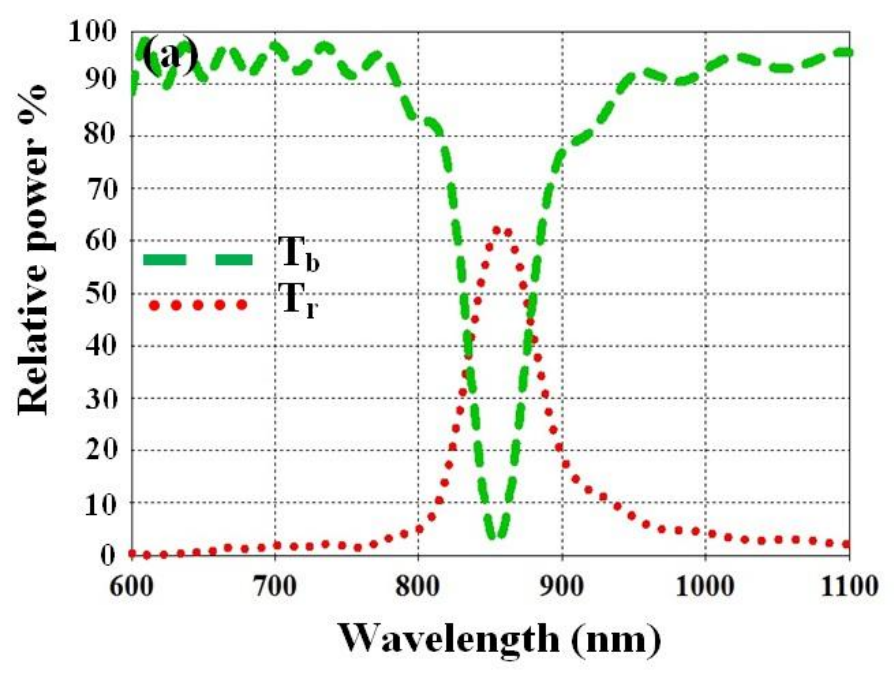




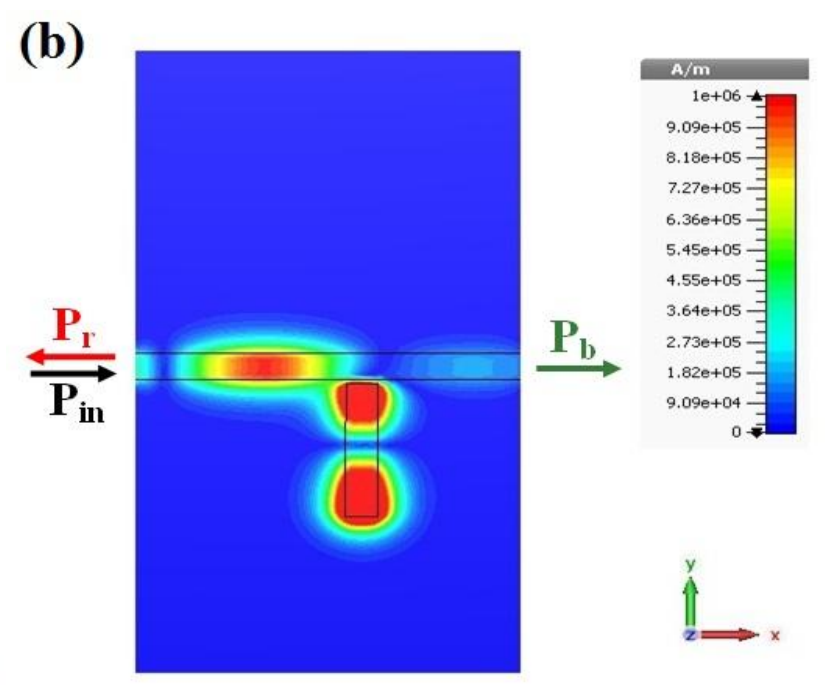

Fig. B-10: (a) Power spectra associated with the cavity 2. (b) field distribution of $\left|\mathrm{H}_{z}\right|$ with $850 \mathrm{~nm}$-incident wavelength.

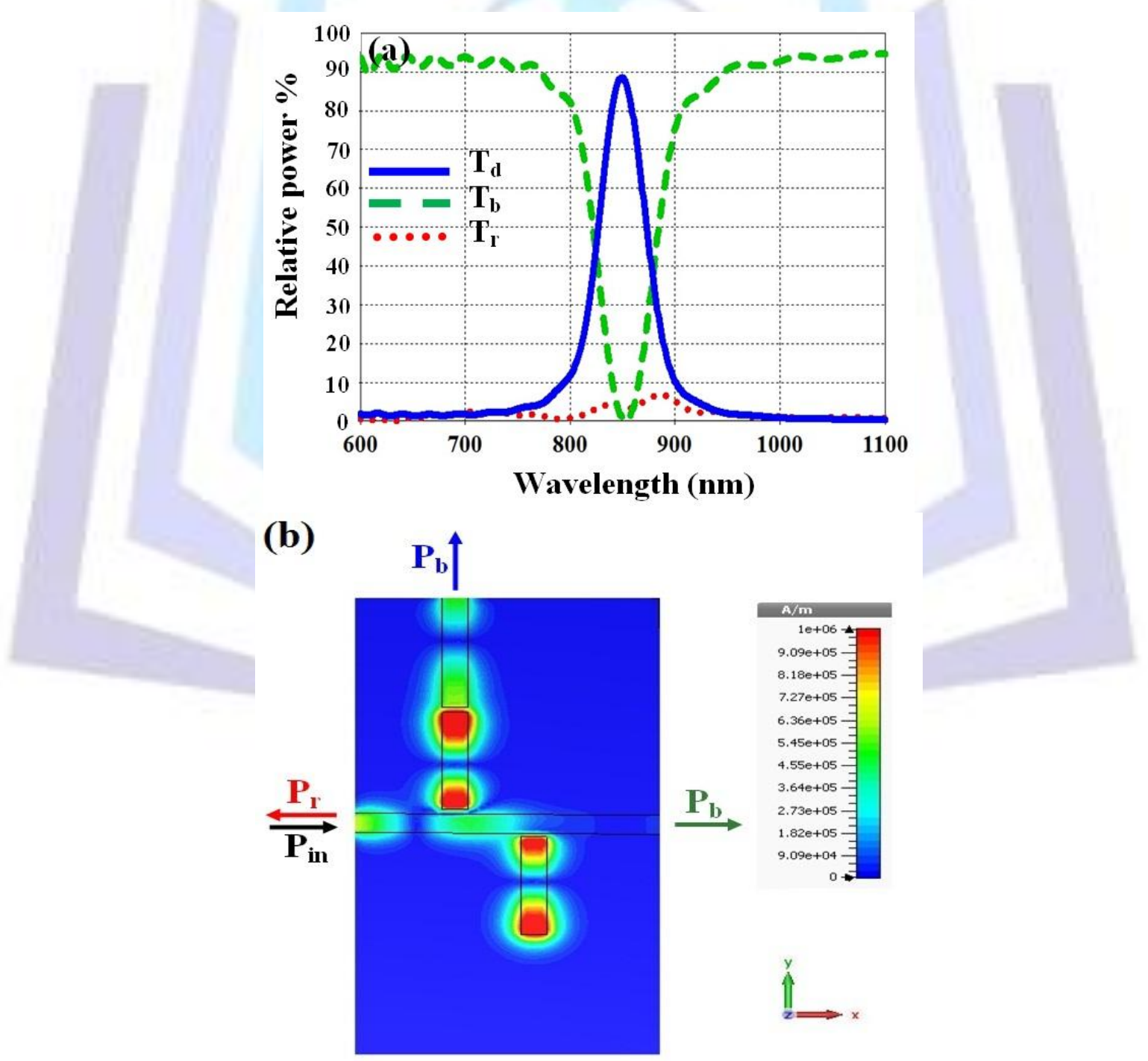

Fig. B-11: Power spectra associated with the $850 \mathrm{~nm}$ demultiplexer. (b) field distribution of $\left|\mathrm{H}_{z}\right|$ with incident wavelength $850 \mathrm{~nm}$. 


\section{Author' biography}

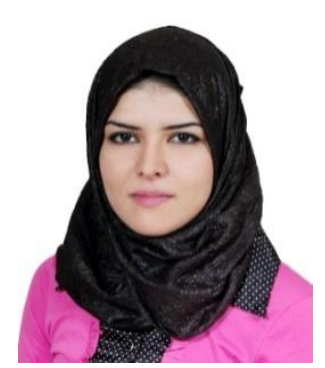

Rasha Hashim Mahdi was born in Baghdad, Iraq, in 1988. She received the B.Sc. degree in Laser and Optoelectronics Engineering from Alnahrain University, Iraq in 2010. Currently, she is a M.Sc. researcher in the Laser and Optoelectronics Engineering Department at Alnahrain University. Her research interests include Plasmonics, WDM, Nanophotonics, Optoelectronics, and Communication systems.

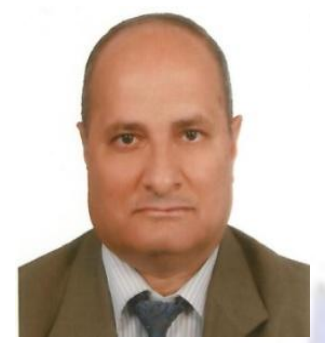

Raad Sami Fyath was born in Maysan, Iraq, in 1954. He received the B.Sc. degree in Electrical Engineering from the University of Basrah, Iraq, in 1976, the M.Sc. degree in Electronics and Communications Engineering from the University of Baghdad, Iraq, in 1987, and the $\mathrm{PhD}$ degree in Electronics Engineering from University of Wales-Bangor, UK, in 1990. Currently, he is a professor of electronics and communications engineering at the College of Engineering, Alnahrain University, Baghdad, Iraq. His research interests include Optical and wireless communications, Optoelectronics, and Nanophotonics. He published more than 100 papers in different scientific journals and conference proceedings.

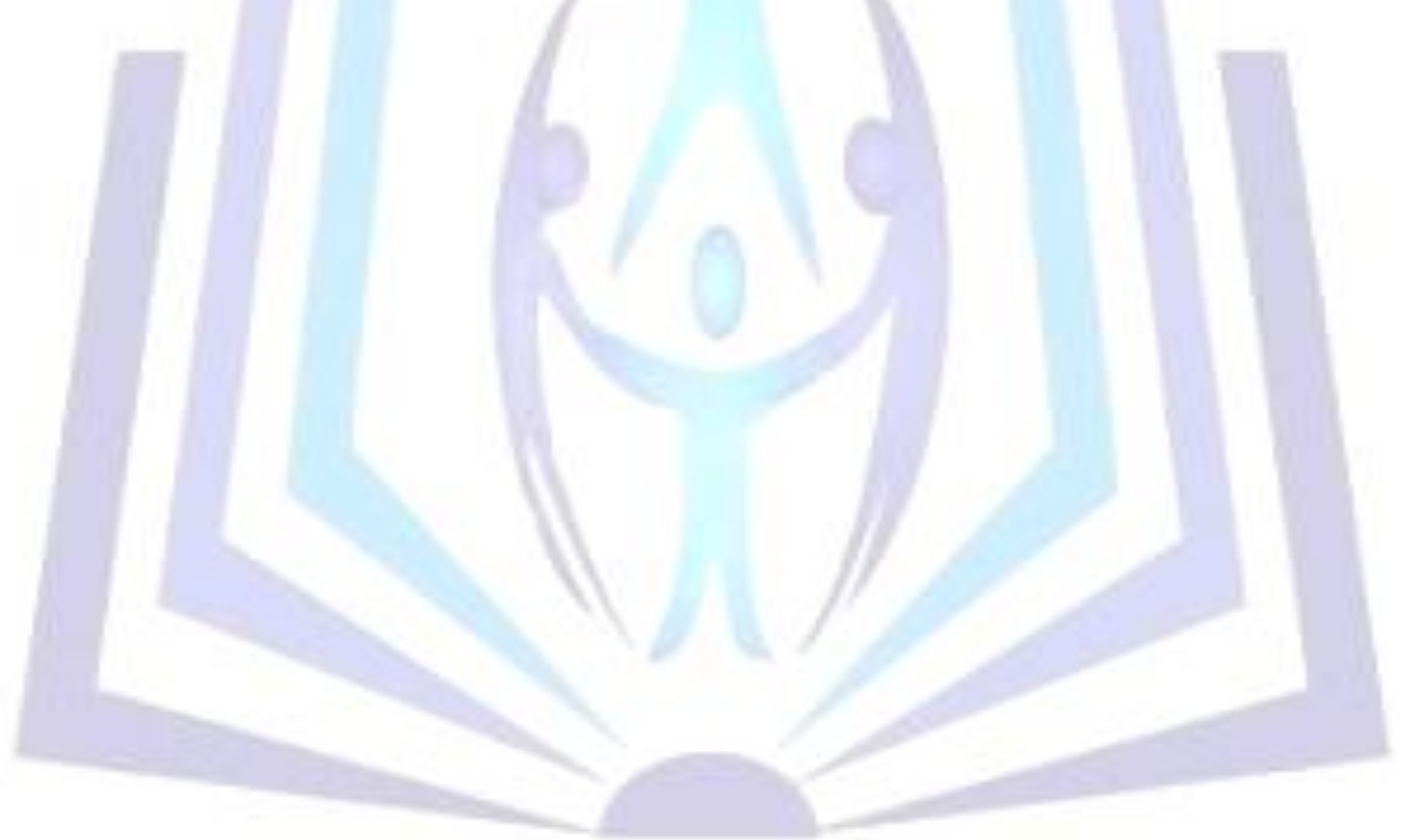

